\title{
Especies arborescentes del género Blechnum (Blechnaceae: Pteridophyta)
}

\section{Cristina H. Rolleri ${ }^{1}$, Carmen Prada ${ }^{2 *}$, José María Gabriel \& Galán ${ }^{3}$ \& Lilian M. Passarelli ${ }^{4}$}

1. Laboratorio de Estudios de Anatomía Vegetal Evolutiva y Sistemática (LEAVES), Facultad de Ciencias Naturales y Museo, 64 entre 120 y diagonal 113, B1904 DZB, Universidad Nacional de La Plata, Argentina. (2) Consejo Nacional de Investigaciones Científicas y Técnicas (CONICET); tinar@speedy.com.ar

2. Departamento de Biología Vegetal I, Facultad de Ciencias Biológicas, Universidad Complutense, Ciudad Universitaria, 28040 Madrid, España; cpm@bio.ucm.es

3. Departamento de Biología Vegetal I, Facultad de Ciencias Biológicas, Universidad Complutense, Ciudad Universitaria, 28040 Madrid, España; jmgabriel@bio.ucm.es

4. Laboratorio de Estudios de Anatomía Vegetal Evolutiva y Sistemática (LEAVES), Facultad de Ciencias Naturales y Museo, 64 entre 120 y diagonal 113, B1904 DZB, Universidad Nacional de La Plata, Argentina; 1mpassarelli@yahoo.com.ar

* Correspondencia.

\section{Recibido 20-I-2012. Corregido 30-IX-2012. Aceptado 30-X-2012.}

\begin{abstract}
Arborescent species of the genus Blechnum (Blechnaceae: Pteridophyta). Although the genus Blechnum has been largely studied, problems of species characterization, nomenclature, classification and taxonomy remain unsolved. For these reasons, in this study we intend to deepen in the characterization of the arborescent group of Blechnum with a review of eight species: B. brasiliense, B. columbiense, B. cycadifolium, B. magellanicum, B. moritzianum, B. schomburgkii, $B$. tabulare and $B$. werckleanum. Type material and several collections from diverse herbaria were studied. For morphological studies we used both herbarium and fresh material collected by the authors in fieldwork between 2005 and 2010. Rhizomes, stipes, vascularization of costae, as well as several characters of the lamina, such as outline, division, venation, margin structure, epidermal patterns, stomata, presence and types of hypodermis, and mesophyll of pinnae were studied. Important aspects of the morphology of the sporophyte have been analyzed, and external characters have been correlated with internal diagnostic ones to use the most reliable external traits in keys and descriptions. Taxonomy has been updated, and corrections have been introduced to the species nomenclature and comments were included to summarize the current knowledge of the geographical distribution and ecology. The presence of B. tabulare in America is confirmed, and the taxonomy of the species is updated, since external and internal morphology indicated that other related, American and African species, such as B. spannagelii and B. madagascariense must be included under that name. Similar studies carried out on B. schomburgkii indicated that the name is a synonym of B. auratum subsp. auratum, while $B$. auratum subsp. columbiense is treated here as a well characterized species. Detailed study of numerous specimens allowed to characterize $B$. moritzianum, being the correct name to apply to the recently described B. yungense. A refreshed description of the species is presented together with a list of new, selected materials to illustrate current known geographical distribution. Expanded data on ecology, geographical distribution and affinities are given, and a key for species identification is presented. Rev. Biol. Trop. 61 (1): 377-408. Epub 2013 March 01.
\end{abstract}

Key words: review, arborescent species, morphology, nomenclature, diagnostic characters.

La bibliografía sobre el género Blechnum L. es muy abundante tanto en lo que respecta a las especies neotropicales como paleotropicales. Sin embargo, aún persisten problemas en la nomenclatura y tipificación de algunas especies, así como la falta de un estudio anatómico completo y una caracterización actualizada de su morfología externa e interna, taxonomía, citología y afinidades, algo que aplica especialmente para varias especies de 
hábito arborescente con escamas rizomáticas aciculares. Por esta razón, se han seleccionado para una caracterización y revisión, ocho especies del género: $B$. brasiliense Desv., $B$. columbiense Hieron., B. cycadifolium (Colla) Sturm, B. magellanicum (Kaulf.) Mett., $B$. moritzianum (Klotzsch) Moore, B. schomburgkii (Klotzsch) C. Chr., B. tabulare (Thunb.) Kuhn y B. werckleanum (H. Christ) C. Chr., algunas poco conocidas.

Blechnum auratum subsp. auratum fue el nombre con el que Tryon \& Stolze (1993) recombinaron Lomaria aurata Fée, incluyendo en su sinonimia a $B$. buchtienii Rosenst. En las claves propuestas por esos autores las diferencias de este taxón con B. schomburgkii no estaban establecidas con suficiente claridad. Blechnum columbiense, descrita por Hieronymus (1908) fue también recombinada como una subespecie de B. auratum (Fée) Tryon y Stolze (Tryon \& Stolze, 1993), pero por el conjunto de sus caracteres se la trata aquí con rango de especie.

Blechnum cycadifolium es una planta del Archipiélago de Juan Fernández, que por su distribución se considera endémica y no hay estudios recientes sobre la especie; convive en ambiente insular con B. magellanicum, que también crece en la patagonia argentino-chilena.

La especie Blechnum moritzianum, basada en Lomaria moritziana Klotzsch, es el nombre que debe aplicarse al material descrito como B. yungense Ramos Giacosa (Ramos Giacosa, 2010). Aquí se la estudia en detalle, se analizan sus afinidades y diferencias con $B$. werckleanum (H. Christ) C. Chr., descrita sobre material de Costa Rica y con B. schomburgkii. Las tres especies tienen una distribución amplia en los Andes sudamericanos y tanto B. moritzianum como $B$. werckleanum presentan un área geográfica extensa no citada previamente.

Blechnum schomburgkii y B. tabulare son exhaustivamente analizados aquí en materiales de toda su área. Blechnum schomburgkii se considera coespecífica de B. auratum subsp. auratum y de $B$. buchtienii, y el nombre que tiene prioridad es el basado en Lomaria schomburgkii Klotzsch. Se analizan su variación, sus afinidades y su área geográfica preferentemente andina.

Blechnum tabulare se revisa en relación con la confirmación de su presencia en Sudamérica, ya citada previamente (Rolleri et al. 2008, Passarelli et al. 2010) y de este análisis se desprende que es coespecífica de $B$. madagascariense y $B$. spannagelii, dos especies poco estudiadas en obras recientes. También se analizan su variación, sus afinidades y su área geográfica, que en este caso incluye localidades selváticas, bordes de selva, áreas de bañados y pajonales inundados en áreas de selvas lluviosas de Brasil, Paraguay, Bolivia y en las selvas subtropicales del NO y NE de la Argentina.

Los caracteres analizados fueron rizomas, estípites y costas, en éstas especialmente la estructura interna y vascularización, caracteres de las láminas como contorno, división, presencia o ausencia de pinnas reducidas, aflebias o aguijones esclerosados procedentes de la atrofia temprana de aquéllas, estructura de los márgenes, venación, modelos epidérmicos, presencia y tipos de hipodermis, mesofilo e indusios.

Los caracteres de la hipodermis, una zona dermatoide adaxial, de espesor variable, que se distingue de la epidermis y del mesofilo en todas las especies estudiadas se describen e ilustran por primera vez. Los análisis de la hipodermis son novedosos para estas especies ya que los previos se refieren en general a gimnospermas y angiospermas (Metcalfe \& Chalk 1950, Esau 1953). Payne \& Peterson (1973) son los únicos autores que integraron, en un estudio comparativo, la descripción de las hipodermis presentes en helechos leptosporangiados, con una actualización de las propuestas de autores previos sobre su origen, como Foster (1936) y Bower (1923).

En trabajos anteriores sobre el género Blechnum (Rolleri \& Prada 2006a, Prada et al. 2008, Rolleri et al. 2008, Gabriel y Galán et al. 2008) se han analizado varios de los rasgos citados para diferentes especies y se ha comprobado su valor diagnóstico en la caracterización de los taxones y en la evaluación de sus afinidades. El alcance del valor diagnóstico de ciertos rasgos externos se puede certificar a 
través del conocimiento de su correlación con algunos caracteres anatómicos de fácil acceso.

Los estudios palinológicos amplios también son recientes en el género y escasos para estas especies. Tryon \& Lugardon (1990) ilustraron 39 especies de todo el género, incluyendo en esa obra las de varias arborescentes; Ramos Giacosa et al. (2009) analizaron con microscopía electrónica de transmisión, entre otras especies del Noroeste de Argentina, las esporas de B. tabulare. Passarelli et al. (2010) efectuaron un análisis amplio utilizando microscopía electrónica de barrido, de 64 especies neotropicales y paleotropicales de Blechnum, incluyendo las de las especies arborescentes estudiadas aquí, proponiendo tipos morfológicos esporales y mostrando la importancia que tiene la ornamentación del perisporio en la caracterización de especies y grupos de afinidad intragenéricos $\mathrm{y}$ con otros géneros de Blechnaceae.

La mayoría de los taxones seleccionados fueron tratados en floras y catálogos, con rango taxonómico de especies o subespecies, por Hieronymus (1908), Schelpe (1952), Stolze (1981), Tryon \& Tryon (1982), Tryon \& Stolze (1993), Moran (1995), Rodríguez Ríos (1995), Dittrich (2005) y Rolleri \& Prada (2006b).

Tryon \& Tryon (1982) propusieron subdividir el género en siete grupos informales basados en los caracteres de los ejes y las frondas, aporte que suministró un primer paso en el ordenamiento de las especies, pero los grupos establecidos resultaron insuficientes o incompletos (Rolleri \& Prada 2006a). De acuerdo con esos autores, algunas especies arborescentes y subarborescentes con escamas lineares fueron reunidas en el grupo $B$. buchtienii, que incluyó B. brasiliense, B. imperiale (Fée \& Glaziou) H. Christ, B. insularum C. V. Morton \& Lellinger, B. schomburgkii, B. spannagelii, B. underwoodianum (Broadh.) C. Chr., B. tabulare y $B$. werckleanum. Blechnum cycadifolium y $B$. magellanicum no se mencionaron entonces, aunque su fenotipo sugería su pertenencia al grupo. Dittrich (2005) renombró algunos grupos según la especie más antigua y reemplazó la denominación de grupo $B$. buchtienii por grupo $B$. tabulare para las especies arborescentes, de las que sólo trató B. schomburgkii y $B$. spannagelii.

En este trabajo se proporcionan nuevos caracteres diagnósticos del esporófito y se revisan otros utilizados previamente con el fin de ponerlos a prueba en la caracterización de los taxones. Los nombres de algunas especies arborescentes se confunden frecuentemente en el material de herbario y esa es otra razón para revisar rasgos externos e internos, fácilmente ponderables en laboratorio, que conduzcan a su mejor determinación. Se elaboran descripciones detalladas, con una actualización de la nomenclatura, tipificación y sinonimia, nuevos datos sobre ecología, distribución geográfica, aspectos adaptativos, afinidades de las especies y se propone una clave para la determinación de las especies.

\section{MATERIALES Y MÉTODOS}

Se estudiaron colecciones del Herbario Nacional Colombiano, del Instituto de Ciencias Naturales de la Universidad Nacional de Colombia (COL), del Museo de La Plata (LP), Instituto-Fundación Miguel Lillo (LIL), Real Jardín Botánico de Madrid (MA), University Herbarium, University of California, Berkeley (UC), United States National Herbarium, Smithsonian Institution (US), Universidad Nacional Mayor de San Marcos (USM) y los tipos relacionados con especies arborescentes existentes en otros herbarios, tales como el herbario de Berlín (Botanic Garden and Botanical Museum Berlin-Dahlem, Freie Universität Berlin, B) y el Herbario de París, Museo Nacional de Historia Natural (P).

Las especies tratadas se citan con los basónimos y sinónimos correspondientes, se hacen comentarios en forma de observaciones, se resumen los datos actualizados de la distribución geográfica, aspectos ecológicos y se da una lista actualizada del material de respaldo seleccionado. En Blechnum magellanicum y B. tabulare, los sinónimos están en Rolleri et al. (2008) y sólo se incluyen sinónimos nuevos derivados de este estudio. Los materiales de referencia de ambas especies son nuevos 
y deben adicionarse a los previamente citados (Rolleri et al. 2008).

Para estudios morfológicos se utilizó material de herbario y recolectado por los autores en trabajos de campo efectuados entre 2005 y 2010. El material de herbario se restauró con una mezcla de butil cellosolve y agua 1:4. Los fragmentos de pinnas se aclararon con hidróxido de sodio al $6 \%$ y se colorearon con azul de toluidina (TBO) al 1\% acuoso. La venación y los modelos epidérmicos se analizaron en pinnas basales, medias y apicales, pero se ilustran sólo la de las pinnas medias. Las dimensiones y densidad de los estomas se tomaron en pinnas medias de todos los ejemplares estudiados; las dimensiones se expresan en largo $\mathrm{x}$ ancho, en $\mu \mathrm{m}$ y la densidad en número de estomas por $\mathrm{mm}^{2}$. Los valores dados representan el promedio de 25 medidas por muestra. Estos datos se incluyen en el Cuadro 1. Toda la terminología relacionada con el esporófito se utiliza según Lellinger (2002). Se priorizan los términos pteridológicos sobre los angiospérmicos, tal como se explica en esa obra (Lellinger 2002), y se prefiere por lo tanto, rizoma, estípite y fronda en lugar de tallo, pecíolo y hoja. El término arborescente se utiliza tal como se indica en Font Quer (1982) y en Lellinger (2002), y no implica la existencia de un hábito arbóreo, derivado de la actividad cambial, sino que hace referencia al rizoma erecto y robusto que generalmente presentan estas especies. Los vocablos relacionados con la epidermis han sido utilizados por los autores en trabajos pteridológicos previos, como Prada et al. (2008) y Prada \& Rolleri (2005) y la relacionada con los estomas se ha tomado de Prabhakar (2003).

\section{RESULTADOS}

En las especies estudiadas las plantas tienen gran porte y alcanzan una altura que va desde 180 hasta $300 \mathrm{~cm}$. Los rizomas son erguidos, desde $50-70$ hasta $120 \mathrm{~cm}$ o más en plantas adultas. Las plantas jóvenes suelen tener hábito arrosetado, pero aún así desarrollan una corona de frondas de hasta $180 \mathrm{~cm}$. El diámetro de los rizomas adultos es de $20 \mathrm{~cm}$ o más; carecen de estolones y son escamosos.

Las escamas del rizoma son lineares, castañas, más o menos rígidas cuando secas y en las partes viejas del rizoma, concoloras o discoloras, con una zona central pluriestratificada que se torna de color castaño más oscuro que el resto y puede esclerosarse y con márgenes hialinos angostos o anchos, más claros, uniestratificados, a veces algo irregulares o repandos, hasta $6 \mathrm{~cm}$ de largo según las especies; se disponen cubriendo densamente el rizoma y la base de los estípites, tienden a curvarse todas en la misma dirección en plantas adultas de algunas especies como $B$. columbiense, $B$. moritzianum, $B$. schomburgkii o bien son más rectas y erguidas, algo retorcidas, como en $B$. brasiliense y B. magellanicum.

Las frondas son monomorfas en B. brasiliense y $B$. columbiense, y dimorfas en las especies restantes. Los estípites nacen más o menos aglomerados, abundantes o escasos, erguidos a divergentes, con un surco adaxial profundo flanqueado por dos zonas elevadas que corresponden a prominencias longitudinales más o menos afiladas, apenas salientes en B. columbiense y extremadamente sobresalientes y curvas en otras especies, como B. cycadifolium, $B$. magellanicum y $B$. schomburgkii, mientras que en la porción abaxial son curvos. En general son rígidos y duros, debido a una zona fibrosa de varias capas de espesor, presente por debajo de la epidermis, que es papilosa; internamente son dictiostélicos, con 2-4 haces adaxiales más grandes que los abaxiales, éstos en un número variable de meristelas desde 5-6 hasta 10 .

Los raquis son lisos y glabros o escamosos, pero en general, esta condición es irregular porque en frondas adultas las escamas suelen ser caducas.

Las costas son marcadamente carenadas en la cara abaxial en B. brasiliense, B. columbiense, B. cycadifolium y B. schomburgkii, moderadamente curvas en $B$. magellanicum, B. moritzianum, B. tabulare y $B$. werckleanum y con un surco adaxial que es más profundo en las especies con costas carenadas, con excepción de B. brasiliense (Fig. 1A-B). Son 


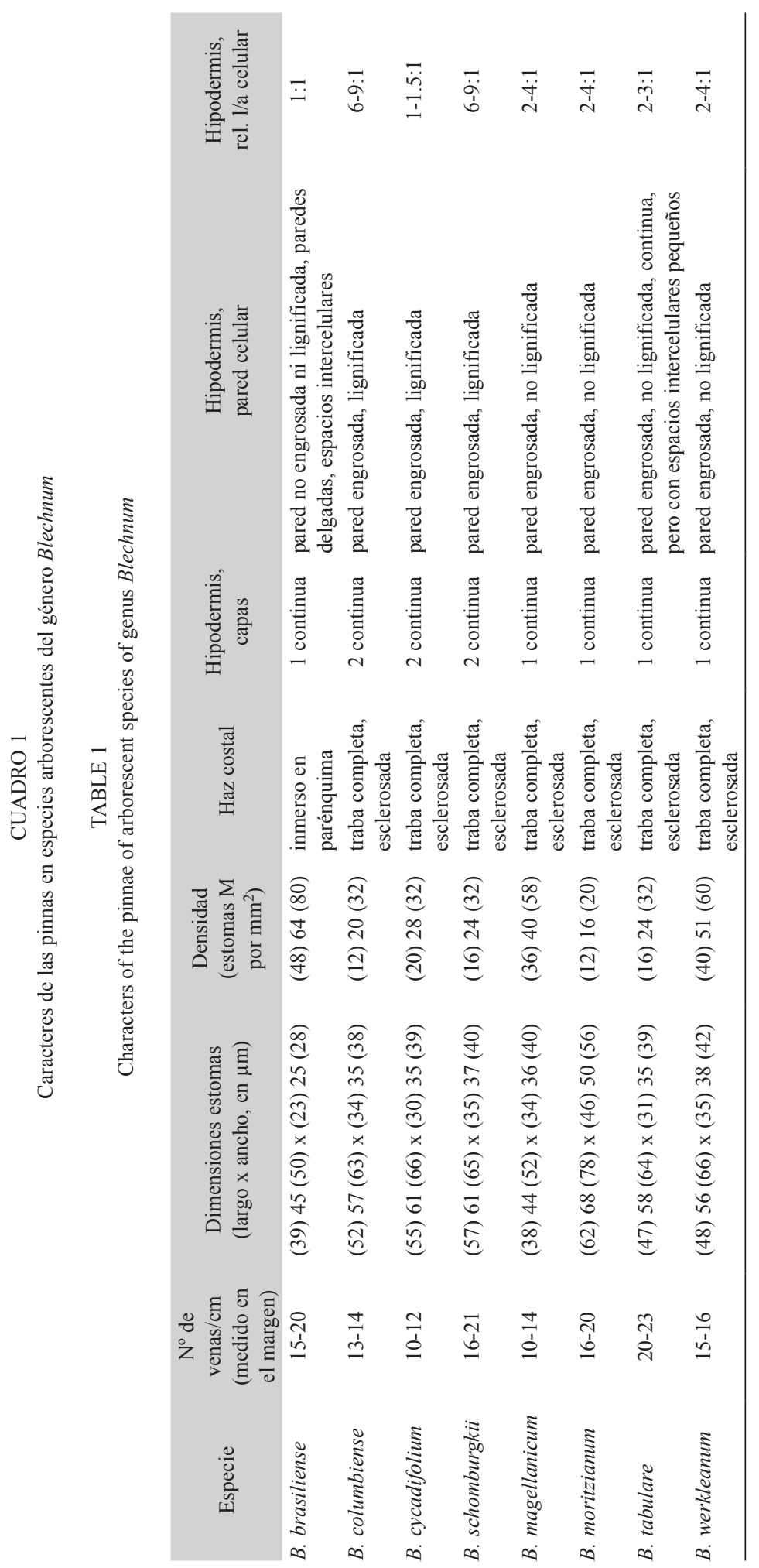



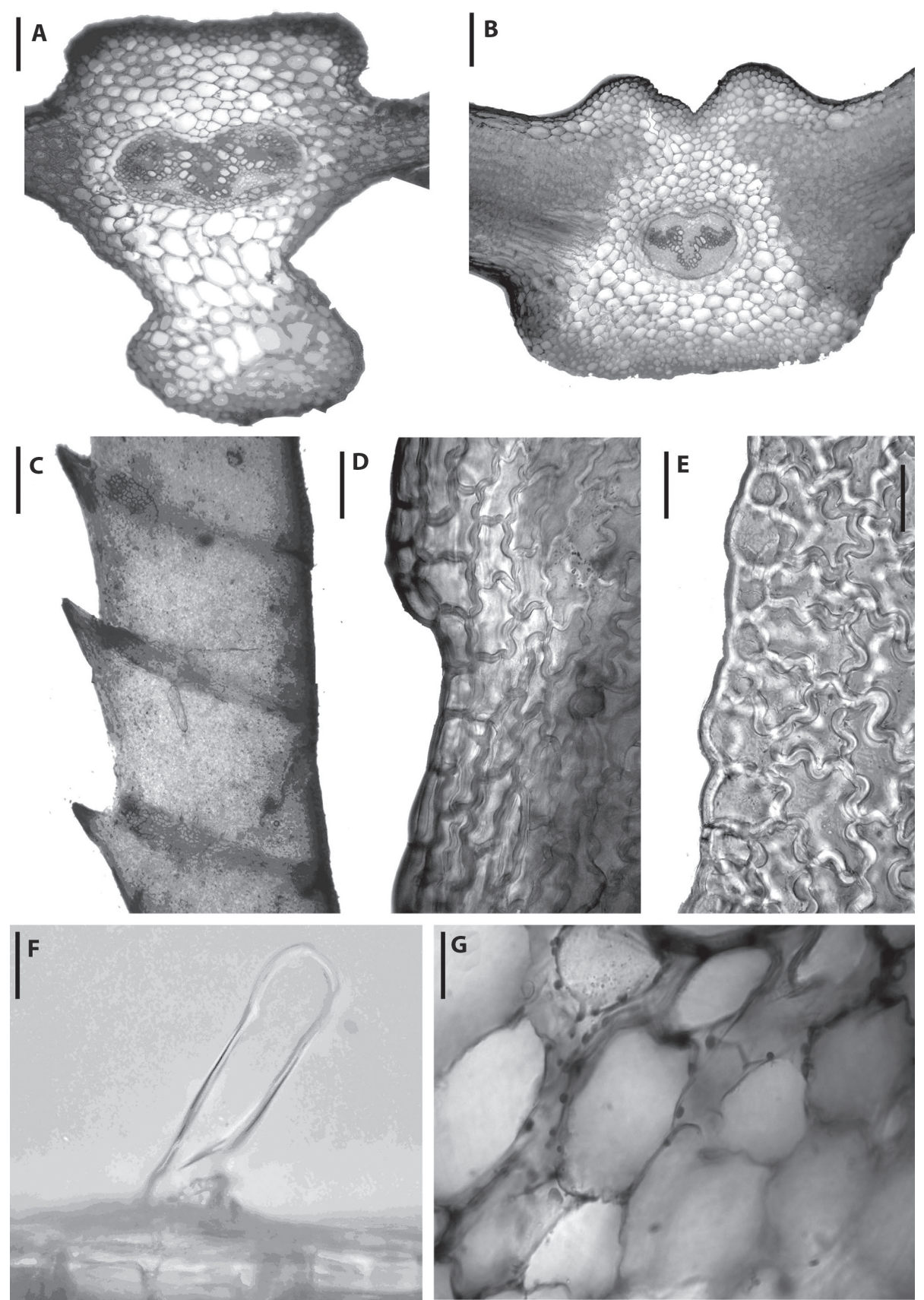

Fig. 1. Anatomía de las pinnas en especies arborescentes de Blechnum. A: sección transversal de la costa en B. brasiliense. B: sección transversal de la costa en $B$. schomburgkii. C-E: márgenes de las pinnas. C: $B$. brasiliense. D-E: $B$. tabulare. F: glándula de mucílago en el raquis de $B$. tabulare. G: protuberancias intercelulares pécticas en parénquima costal de $B$. brasiliense. Escala: $200 \mu \mathrm{m}$ en A-B, $0.5 \mathrm{~mm}$ en $\mathrm{C}, 50 \mu \mathrm{m}$ en $\mathrm{D}-\mathrm{G}$.

Fig. 1. Anatomy of the pinnae in arborescent species of Blechnum. A: Cross section of the costa in B. brasiliense. B: Cross section of the costa in B. schomburgkii. C-E: Margins of the pinnae. C: B. brasiliense. D-E: B. tabulare. F: Mucilage gland on the rachis of $B$. tabulare. G: Intercellular pectic protuberances in costal parenchyma of B. brasiliense. Scale: $200 \mu \mathrm{m}$ in A-B, $0.5 \mathrm{~mm}$ in $\mathrm{C}, 50 \mu \mathrm{m}$ in D-G. 
glabrescentes y abaxialmente pueden ser escamosas, pero la densidad del indumento varía ya que las escamas son caducas. En B. magellanicum y $B$. tabulare se presentan pequeñas glándulas de mucílago 1-celulares, largamente persistentes aún en material herborizado (Fig. 1F). En sección transversal presentan un solo haz, con xilema en forma de $\mathrm{V}$ de brazos divergentes, rodeado por floema, parénquima y una endodermis con bandas de Caspary o engrosamientos en forma de U. Los haces en V están trabados por tejido esclerosado que se desarrolla hacia ambas epidermis, abaxial y adaxial, en todas las especies (Fig. 1B) con excepción de $B$. brasiliense, en la que están inmersos en parénquima laxo, sin tejido esclerosado asociado (Fig. 1A). En esta última el parénquima del área costal está constituido por grandes células en cuyas paredes primarias celulósicas se encuentran protuberancias intercelulares pécticas en forma de verrugas claramente visibles (Fig. 1G). Ocasionalmente en pinnas jóvenes de $B$. werckleanum se ha observado que los haces presentan una traba esclerosada parcial hacia el hipofilo y un área en forma de casquete esclerosado hacia el epifilo (Cuadro 1).

La forma y división de las láminas es variable en las especies. En B. brasiliense el contorno es oblanceolado a espatulado (Fig. 2A), con ápice obtuso y base atenuada, muy gradualmente debido a la presencia de pinnas cuya lámina se reduce más y más basípetamente, hasta formar pequeñas aurículas planas, herbáceas o papiráceas, según la edad de la fronda. La lámina es pinnatífida, con pinnas adnatas planas cuyas bases surcurrentes y decurrentes se unen. El estípite es muy corto a casi inexistente.

En B. moritzianum la lámina es lanceolado elíptica (Fig. 2C) y en B. werckleanum es oblonga (Fig. 2B), en ambos casos con ápices agudos y bases abruptamente reducidas; en general sólo uno o dos pares basales de pinnas son algo más cortos que los restantes. Las láminas son pinnadas en ambas especies, con pinnas planas distanciadas, que son horizontales, lanceoladas y con ápices obtusos a obtusomucronados en B. moritzianum (Fig. 3G) y ascendentes, angostamente oblongas y con ápices caudados, curvados acroscópicamente y algo aserrados en $B$. werckleanum (Fig. $3 \mathrm{H}$ ). Los estípites representan $1 / 3$ o más de la longitud de la lámina.

En B. columbiense (Fig. 2D), B. cycadifolium (Fig. 2F), B. magellanicum (Fig. 2E) y B. schomburgkii (Fig. 2G) las láminas tienen contorno oblanceolado elíptico. En las tres primeras las bases son reducidas más bien abruptamente; en B. cycadifolium y B. magellanicum pueden verse 2-3 pares de pinnas basales algo más cortas pero raramente aflebias y son frecuentes los aguijones resultantes de la esclerosis temprana de pinnas atrofiadas. Los estípites pueden alcanzar $1 / 3$ de la longitud de la lámina en $B$. columbiense y son más cortos en $B$. cycadifolium y $B$. magellanicum. En $B$. columbiense y $B$. cycadifolium la fronda es pinnada: en $B$. columbiense con pinnas lineares divergentes o ascendentes, cuyas láminas están marcadamente enrolladas hacia la cara abaxial, tienen ápices romos enrollados y son sub-pecioluladas (Fig. 3A), mientras que en B. cycadifolium las pinnas son lanceoladas con láminas planas pero con los márgenes revolutos, ápices agudos rígidos y bases sésiles (Fig. 3E). En B. magellanicum la lámina es pinnatífida a pinnada, con segmentos adnatos en el tercio apical o hasta la mitad de la lámina, luego pinnada y con pinnas sésiles planas ascendentes y ápices agudos atenuados.

El contorno es también oblanceolado a elíptico en B. schomburgkii, con bases redondeadas, muy raramente atenuadas y estípites cortos o largos, sin aflebias; el desarrollo de los estípites varía en la misma planta, pero en general en frondas adultas son más bien largos, alcanzando hasta $1 / 3$ de la longitud de la lámina, que es pinnada; aunque ocasionalmente las pinnas apicales son adnatas, la condición pinnada es la más frecuente, con pinnas ascendentes, lanceolado-lineares a lanceoladas, atenuadas en el ápice y la base, que es sésil, y con los márgenes revolutos prácticamente en toda su extensión (Fig. 3B-D).

En B. tabulare el contorno es elíptico (Fig. 2H-I), la base se atenúa en pares de pinnas gradualmente reducidas hasta auriculiformes, 


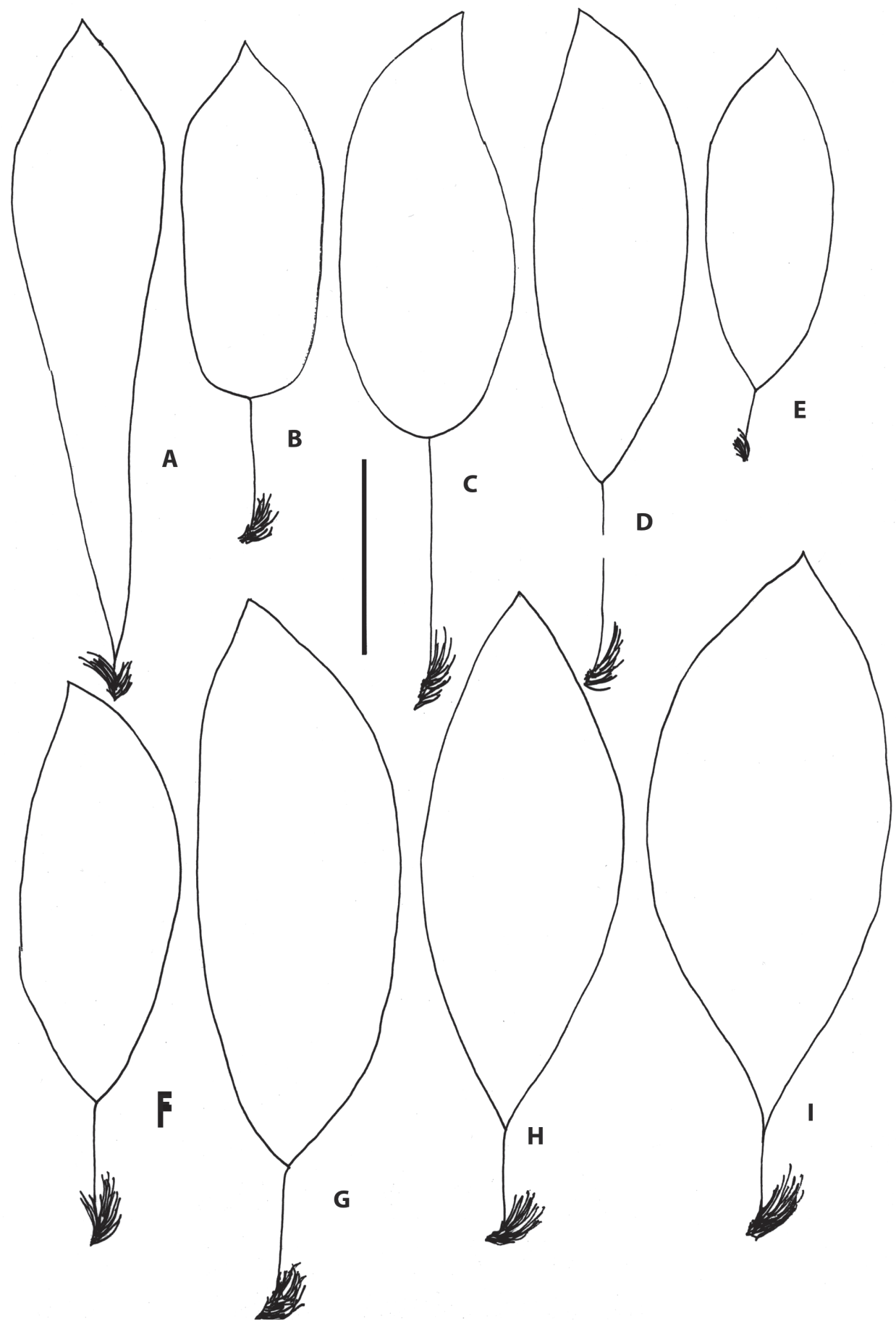

Fig. 2. Contorno de las frondas en especies arborescentes de Blechnum. A: B. brasiliense. B: B. werckleanum. C: B. moritzianum. D: B. columbiense. E: B. magellanicum. F: B. cycadifolium. G: B. schomburgkii. H-I: B. tabulare. Escala: $50 \mathrm{~cm}$.

Fig. 2. Outline of the laminae in fronds of arborescent species of Blechnum. A: B. brasiliense. B: B. werckleanum. C: B. moritzianum. D: B. columbiense. E: B. magellanicum. F: B. cycadifolium. G: B. schomburgkii. H-I: B. tabulare. Scale: $50 \mathrm{~cm}$. 


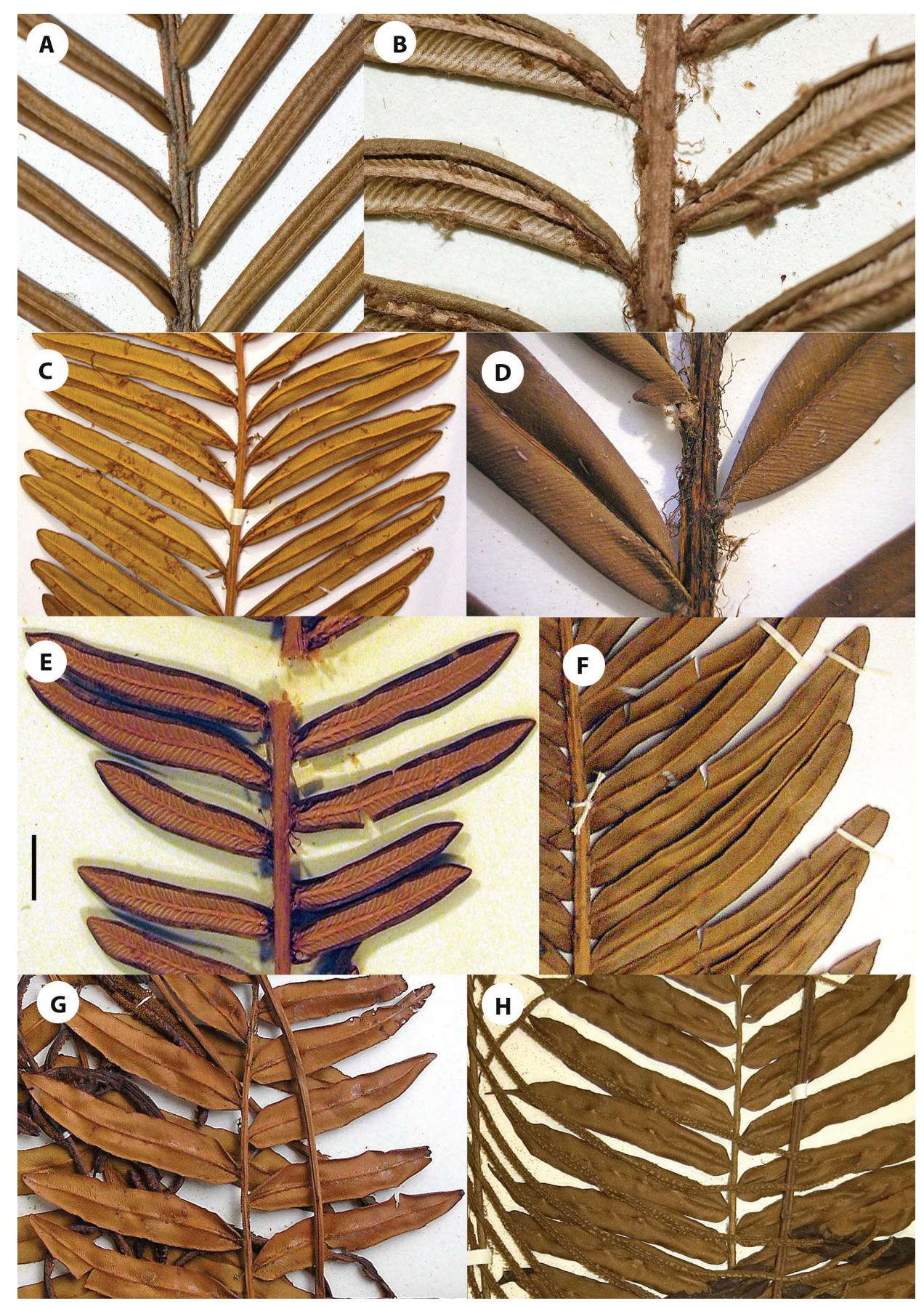

Fig. 3. Morfología externa de las pinnas en especies arborescentes de Blechnum. A: B. columbiense. B-D: B. schomburgkii. E: B. cycadifolium. F: B. tabulare. G: B. moritzianum. H: B. werkleanum. Escala: $0.5 \mathrm{~cm}$ en A- B, D, $1 \mathrm{~cm}$ en C, E, $2 \mathrm{~cm}$ en F, $3 \mathrm{~cm}$ en $\mathrm{G}, \mathrm{H}$.

Fig. 3. External morphology of the pinnae in arborescent species of Blechnum. A: B. columbiense. B-D: B schomburgkii. E: B. cycadifolium. F: B. tabulare. G: B. moritzianum. H: B. werckleanum. Scale: $0.5 \mathrm{~cm}$ in A-B, D, $1 \mathrm{~cm}$ in C, E, F $2 \mathrm{~cm}$, $3 \mathrm{~cm}$ in $\mathrm{G}, \mathrm{H}$. 
planas y no esclerosadas ni transformadas en aguijones; los estípites tienen longitud muy variable, desde tres o cinco $\mathrm{cm}$ hasta $1 / 3 \mathrm{de}$ la lámina; ésta es pinnatífida a pinnada, la adnación de las pinnas puede incluir porciones apicales y basales de la lámina; las pinnas son planas, oblongas angostas, horizontales a ascendentes, con ápices obtusos, a veces mucronados y márgenes también planos (Fig. 3F).

Los márgenes son regularmente aserrados en $B$. brasiliense (Fig. 1C), repandos planos en B. magellanicum, B. moritzianum, B. tabulare (Fig. 1D-E) y B. werckleanum, o repandos revolutos en $B$. columbiense, $B$. cycadifolium y B. schomburgkii.

Las venas son predominantemente simples, con algunas bifurcadas a distintas distancias de la costa en B. brasiliense (Fig. 4E), simples y bifurcadas una vez, casi en origen (geminadas) o cerca de la costa en B. columbiense (Fig. 4A), B. cycadifolium (Fig. 4B), B. magellanicum (Fig. 4C) y B. schomburgkii (Fig. 4D); o bien como las anteriores pero con algunas venas bifurcadas dos veces, la segunda división más próxima al margen, en $B$. moritzianum (Fig. 4F), B. tabulare (Fig. 4H) у $B$. werckleanum (Fig. 4G). La distancia entre las venas es similar en todas las especies excepto en $B$. tabulare, que presenta venas muy próximas con dicotomías de ramas casi paralelas (Cuadro 1).

Los modelos epidérmicos difieren en epifilos e hipofilos. Los epifilos tienen células de paredes casi rectas a levemente sinuosas en B. cycadifolium (Figs. 5A; 8A) y son diversamente sinuosos en las especies restantes. Las ondulaciones son simples, someras, poco frecuentes en B. schomburgkii (Fig. 5D) y $B$. werckleanum (Figs. 6A; 8B) y más frecuentes en B. columbiense (Fig. 5G) y B. magellanicum (Fig. 6D). Las ondas son también simples, pero profundas en B. tabulare (Figs. 6G; 7A; C). Las ondulaciones son compuestas y curvas en B. brasiliense (Fig. 7G) y compuestas, curvas y angulosas, en B. moritzianum (Figs. 7D; 8D). Los hipofilos son menos variables, con ondas simples, generalmente profundas, en $B$. columbiense (Fig. 5I), B. cycadifolium (Fig. 5C), B. magellanicum (Fig. 6F), B. schomburgkii (Fig. 5F), B. tabulare (Figs. 6I; 7C; 8E) y B. werckleanum (Fig. 6C), y compuestas, con engrosamientos en curvas y senos en $B$. brasiliense (Fig. 7I) y B. moritzianum (Figs. 7F; 8F).

Las epidermis tienen células curvas en sección transversal. Los estomas son algo hundidos, diacíticos, anomocíticos, actinocíti$\cos$, y menos frecuentemente, estaurocíticos y tetracíticos. Presentan engrosamientos de pared epidérmica y pared de contacto de oclusivas, de pared poral y piezas polares en T. La pared epidérmica y la pared de contacto de oclusivas, así como la pared poral está muy engrosada en $B$. columbiense, B. cycadifolium, B. magellanicum y $B$. schomburgkii. Las piezas polares en $\mathrm{T}$ se observaron especialmente en B. magellanicum y B. schomburgkii. En las pinnas con láminas más anchas, los estomas se encuentran en 4-6 hileras entre las venas. Las dimensiones y densidades estomáticas se incluyen en el Cuadro 1.

La hipodermis está presente en todas las especies estudiadas, en posición adaxial y es continua. Está constituida por 1-2 capas de células alargadas en el sentido transversal, perpendiculares a la vena y de longitudes variables, equivalentes a 1-2 y hasta 5-6 células epidérmicas suprayacentes. Las relaciones longitud/anchura (1/a) de estas células hipodérmicas van desde 1:1, es decir, casi isodiamétricas, en $B$. brasiliense (Fig. $7 \mathrm{H}$ ) у B. cycadifolium (Fig. 5B), 2-4:1 en B. magellanicum (Fig. 6E), B. moritzianum (Fig. 7E), B. tabulare (Figs. $6 \mathrm{H}$; 7B) y B. werckleanum (Fig. 6B), hasta las células muy largas, con relación 1/a 6-9:1 de $B$. columbiense (Fig. 5H; 9A) y B. schomburgkii (Fig. 5E). En estas tres últimas especies la hipodermis suele formar dos y hasta tres capas de células con paredes marcadamente engrosadas, de contorno rectangular con punteaduras y paredes esclerosadas que reaccionan dando color azul verdoso intenso con TBO (Fig. 9A; C-D). En las especies restantes la hipodermis suele presentar una sola capa, con células cuyas paredes no están lignificadas pero sí variablemente engrosadas (Fig. 9B, E-F), excepto en B. brasiliense, que las presenta con paredes delgadas y casi isodiamétricas, con espacios 


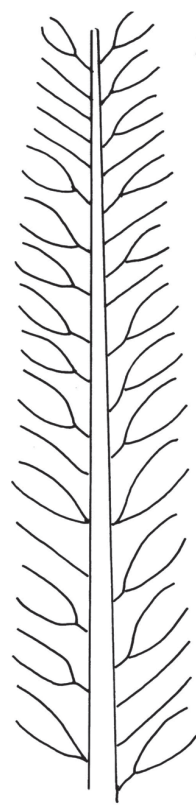

A

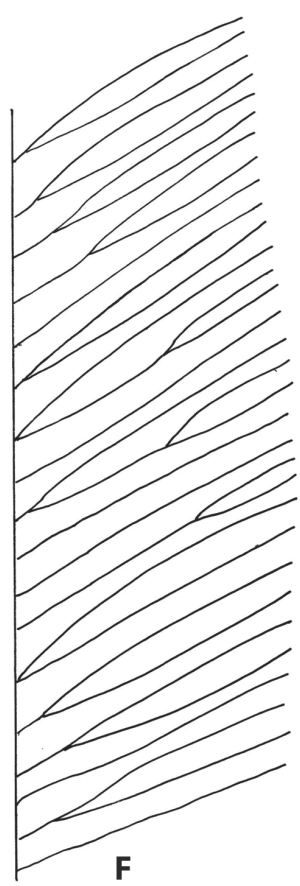

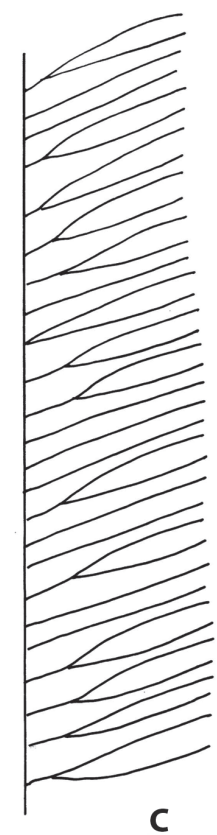

C
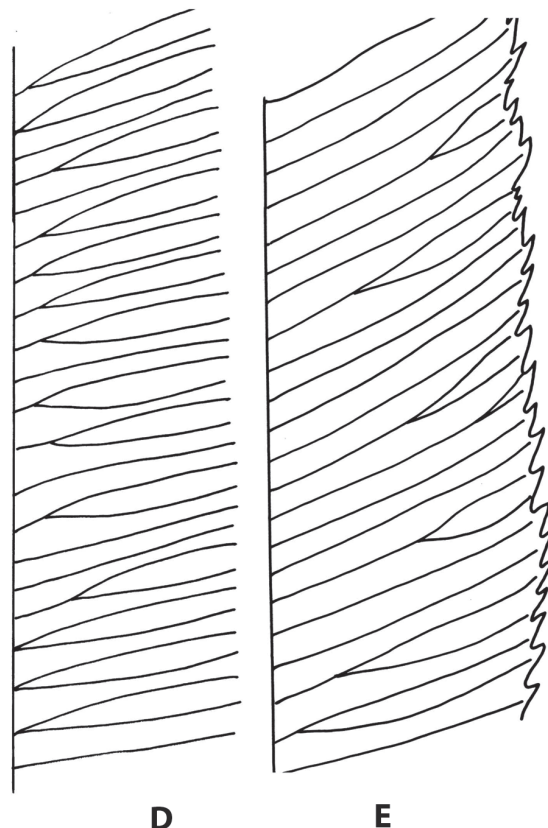

$\mathbf{E}$

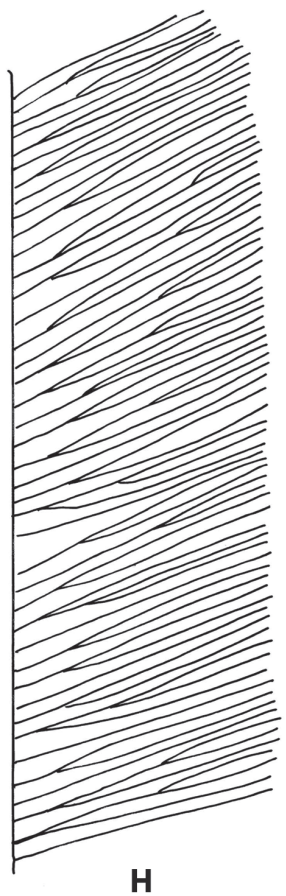

G

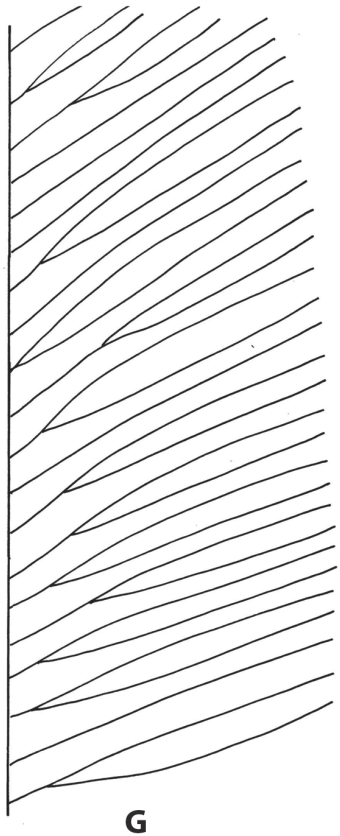

Fig. 4. Patrones de venación en especies arborescentes de Blechnum. A: B. columbiense. B: B. cycadifolium. C: B. magellanicum. D: B. schomburgkii. E: B. brasiliense. F: B. moritzianum. G: B. werckleanum. H: B. tabulare. Escala: $1 \mathrm{~cm}$ en A-D y H, $0.75 \mathrm{~cm}$ en E-G.

Fig. 4. Venation patterns in arborescent species of Blechnum. A: B. columbiense. B: B. cycadifolium. C: B. magellanicum. D: B. schomburgkii. E: B. brasiliense. F: B. moritzianum. G: B. werckleanum. H: B. tabulare. Scale: $1 \mathrm{~cm}$ in A-D and $\mathrm{H}$, $0.75 \mathrm{~cm}$ in $\mathrm{E}-\mathrm{G}$. 

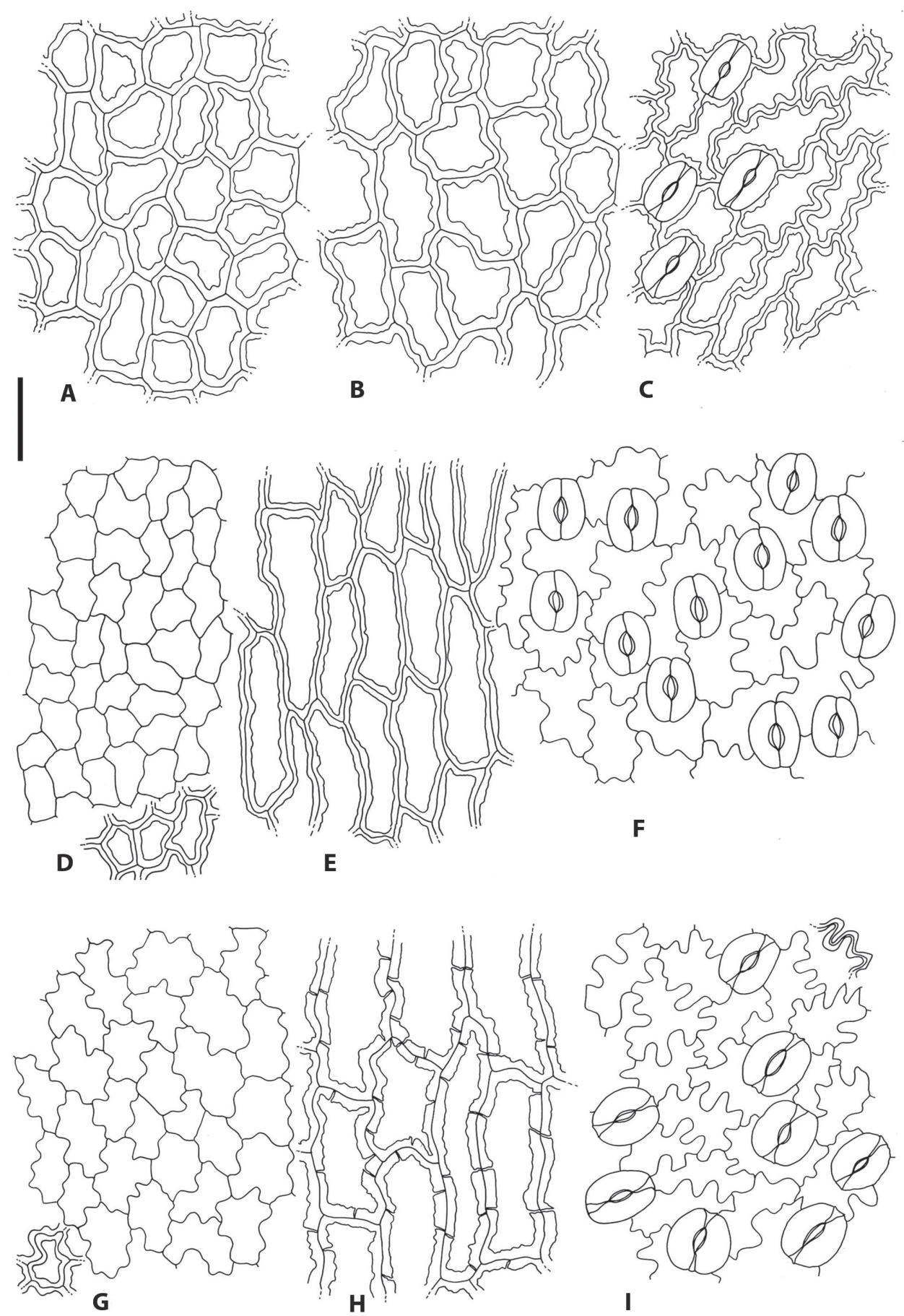

Fig. 5. Modelos epidérmicos en especies arborescentes de Blechnum. A-C: B. cycadifolium. D-F: B. schomburgkii. G-I: B. columbiense. Izquierda, epifilos; derecha hipofilos; centro, hipodermis. Escala: $100 \mu \mathrm{m}$.

Fig. 5. Epidermal patterns in arborescent species of Blechnum. A-C: B. cycadifolium. D-F: B. schomburgkii. G-I: B. columbiense. Left, epiphylls, right, hypophylls, center, hypodermis. Scale: $100 \mu \mathrm{m}$. 

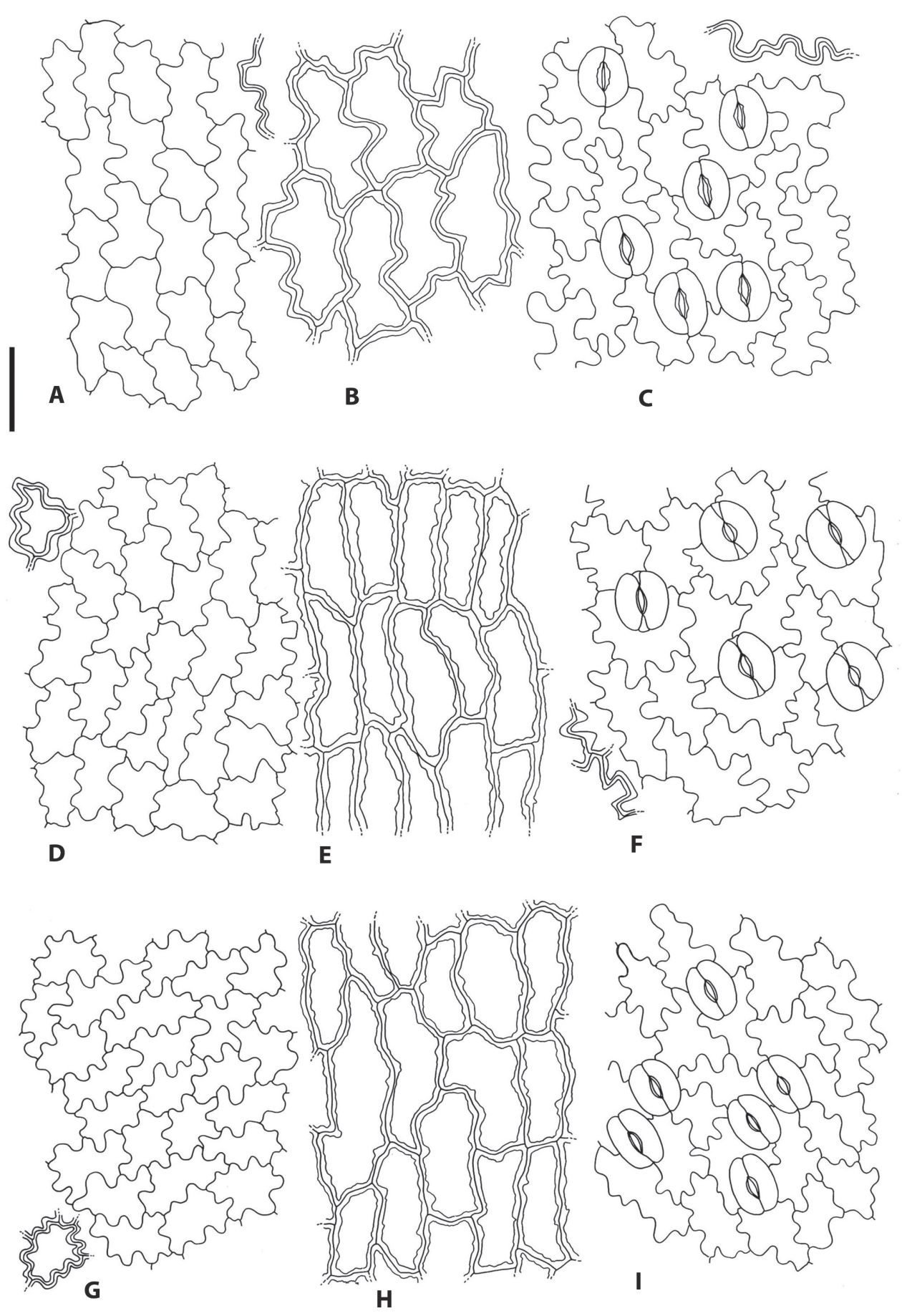

Fig. 6. Modelos epidérmicos en especies arborescentes de Blechnum. A-C: B. werckleanum. D-F: B. magellanicum. G-I: B. tabulare, de África. Izquierda, epifilos; derecha hipofilos; centro, hipodermis. Escala $100 \mu \mathrm{m}$.

Fig. 6. Epidermal patterns in arborescent species of Blechnum. A-C: B. werckleanum. D-F: B. magellanicum. G-I: B. tabulare, from Africa. Left, epiphylls, right, hypophylls, center, hypodermis. Scale: $100 \mu \mathrm{m}$. 

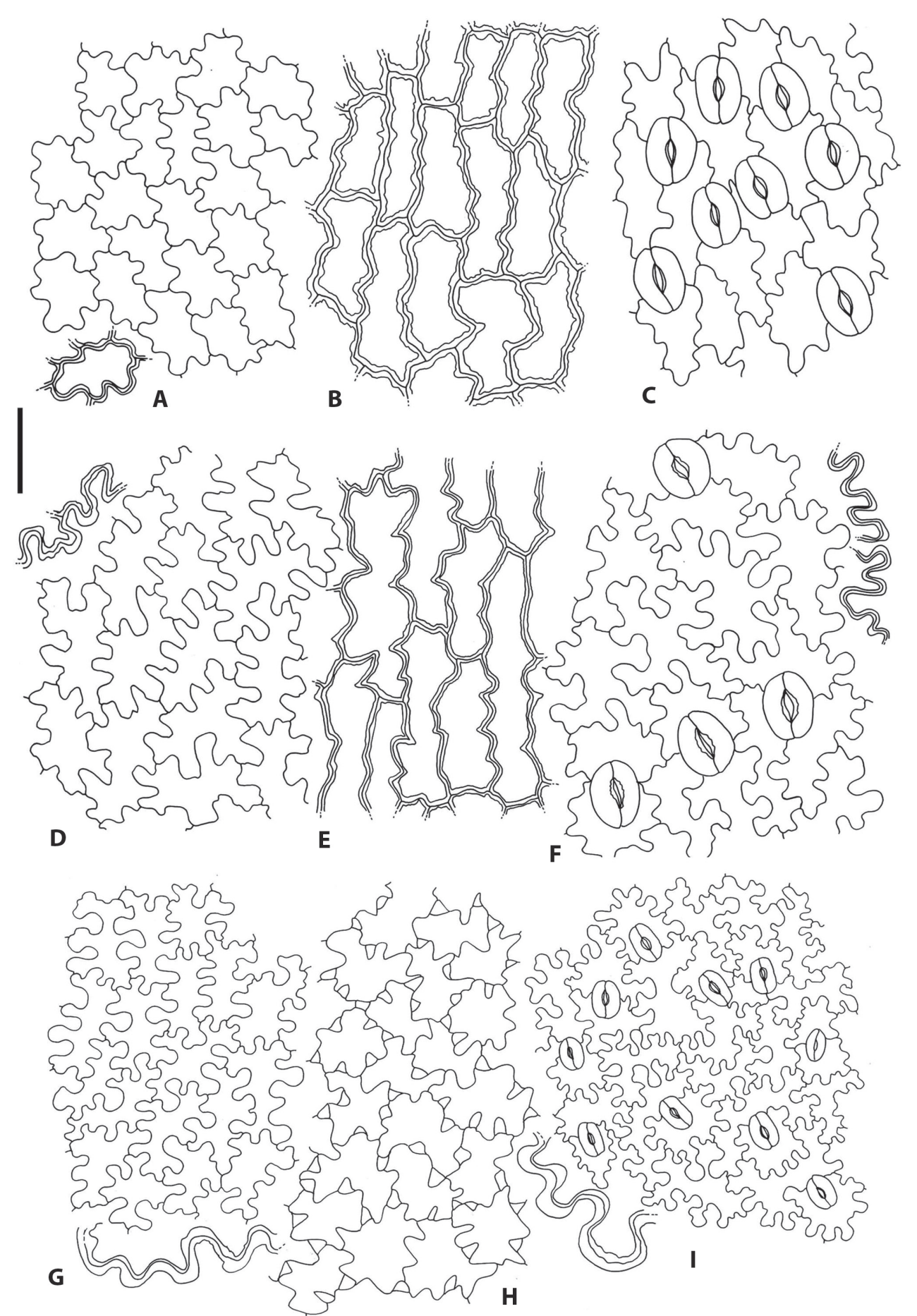

Fig. 7. Modelos epidérmicos en especies arborescentes de Blechnum. A-C: B. tabulare, de América. D-F: B. moritzianum. G-I: B. brasiliense. Izquierda, epifilos; derecha hipofilos; centro, hipodermis. Escala: $100 \mu \mathrm{m}$.

Fig. 7. Epidermal patterns in arborescent species of Blechnum. A-C: B. tabulare, from America. D-F: B. moritzianum. G-I: $B$. brasiliense. Left, epiphylls, right, hypophylls, center, hypodermis. Scale: $100 \mu \mathrm{m}$. 

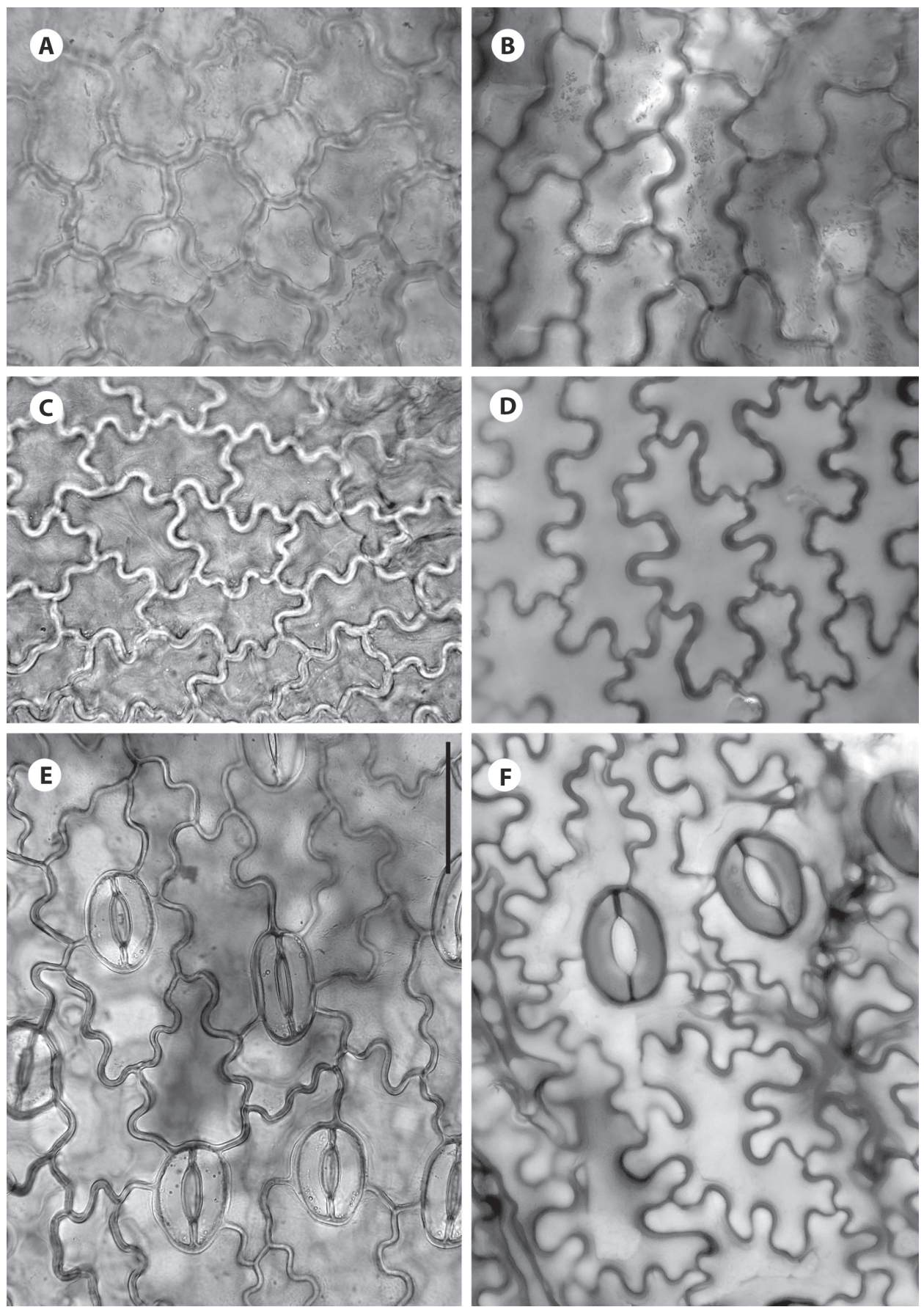

Fig. 8. Modelos epidérmicos en especies arborescentes de Blechnum. A-D: epifilos. A: B. cycadifolium. B: B. werckleanum. C: B. tabulare. D: B. moritzianum. E-F: hipofilos. E: B. tabulare. F: B. moritzianum. Escala: $35 \mu \mathrm{m}$ en A-B, $40 \mu \mathrm{m}$ en C, $85 \mu \mathrm{m}$ en D, $100 \mu \mathrm{m}$ en E-F.

Fig. 8. Epidermal patterns in arborescent species of Blechnum. A-D: Epiphylls. A: B. cycadifolium. B: B. werckleanum. C: B. tabulare. D: B. moritzianum. E-F: Hypophylls. E: B. tabulare. F: B. moritzianum. Scale: $35 \mu \mathrm{m}$ in A-B, $40 \mu \mathrm{m}$ in C, $85 \mu \mathrm{m}$ in $\mathrm{D}, 100 \mu \mathrm{m}$ in $\mathrm{E}-\mathrm{F}$. 

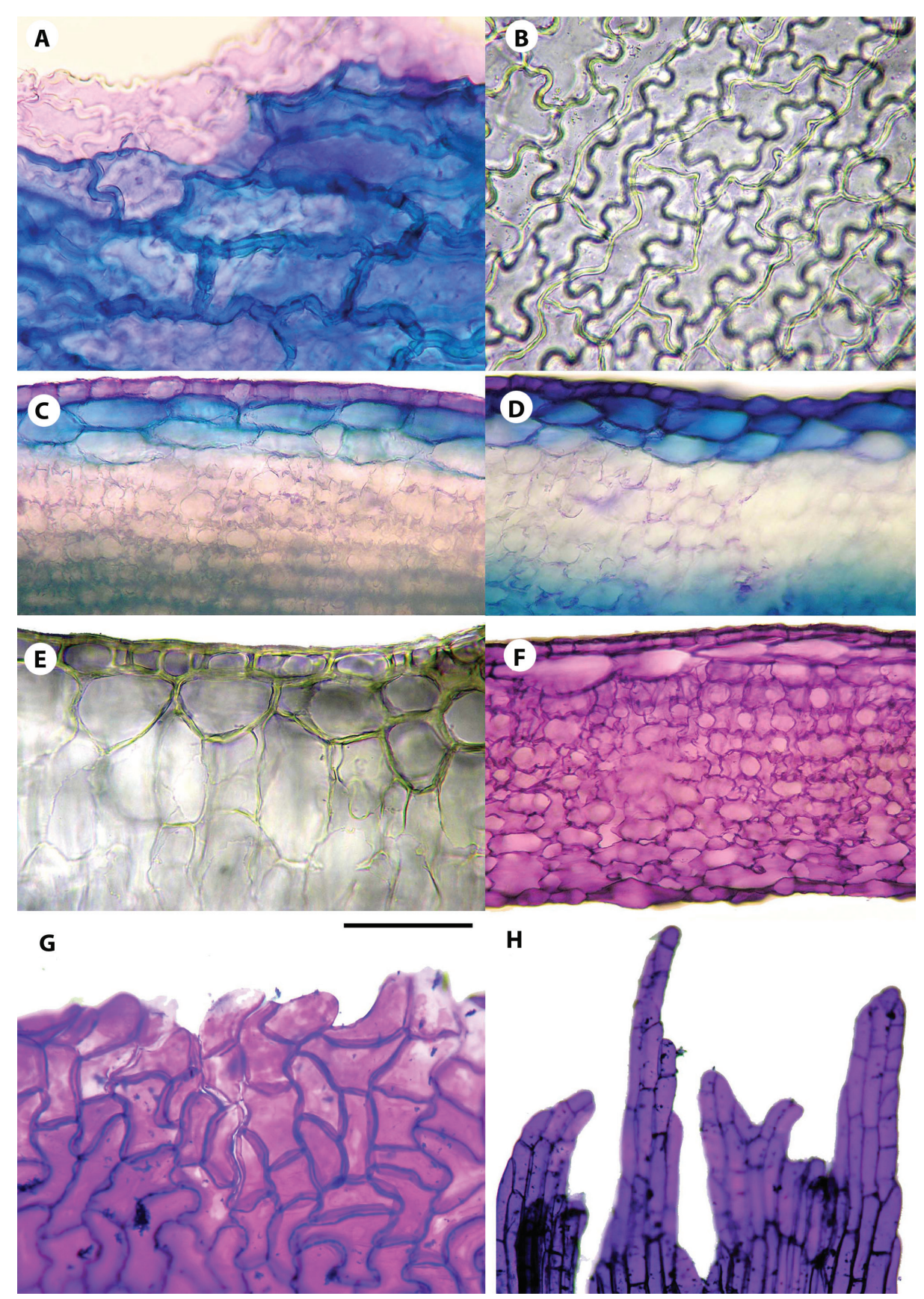

Fig. 9. Hipodermis e indusios en especies arborescentes de Blechnum. A-B: hipodermis en vista superficial. A: $B$. columbiense. B: B. tabulare. C-F: hipodermis en secciones transversales de pinnas. C: B. schomburgkii. D: B. columbiense. E: B. tabulare. F: B: werckleanum. G-H: márgenes de los indusios. G: B. brasiliense. H. B: moritzianum. Escalas: $40 \mu \mathrm{m}$ en A-B; $250 \mu \mathrm{m}$ en $\mathrm{C}-\mathrm{D} ; 125 \mu \mathrm{m}$ en $\mathrm{E} ; 400 \mu \mathrm{m}$ en $\mathrm{F} ; 500 \mu \mathrm{m}$ en G: $250 \mu \mathrm{m}$ en $\mathrm{H}$.

Fig. 9. Hypodermis and indusia in arborescent species of Blechnum. A-B: Hypodermis, superficial view. A: B. columbiense. B: B. tabulare. CF: Hypodermis, in cross sections of pinnae. C: B. schomburgkii. D: B. columbiense. E: B. tabulare. F: B: werckleanum. G-H: Margins of the indusia. G: B. brasiliense. H. B: moritzianum. Scale: $40 \mu \mathrm{m}$ in A-B, $250 \mu \mathrm{m}$ in C-D, $125 \mu \mathrm{m}$ in $\mathrm{E}, 400 \mu \mathrm{m}$ in $\mathrm{F}, 500 \mu \mathrm{m}$ in $\mathrm{G}, 250 \mu \mathrm{m}$ in $\mathrm{H}$. 
intercelulares pequeños (Cuadro 1). En sección transversal las células hipodérmicas se distinguen bien por tener mayor lumen y tamaño que las epidérmicas.

El mesofilo es compacto con excepción del de $B$. brasiliense. Por debajo de la hipodermis se observa parénquima de translocación. En la mayoría de las especies el tejido translocador esponjoso y el clorénquima no muestran una transición morfológica clara entre uno y otro. Hacia la cara adaxial se puede presentar un clorénquima laxo, formado por células con forma de horqueta y dispuestas con espacios intercelulares amplios, como en $B$. brasiliense y $B$. moritzianum, o con aspecto homogéneo, con espacios intercelulares más reducidos, como en las especies restantes.
Los indusios son anchos, alcanzan los 4-5mm, se curvan, tiene márgenes repandos denticulados en B. brasiliense (Fig. 9G) y en el resto de las especies se endurecen marcadamente en pinnas fértiles adultas y tienen márgenes irregulares (Fig. 9H); están diferenciados en dos porciones: la proximal a su inserción constituida por 1-2 (3) estratos celulares con modelos epidérmicos poligonales o sinuosos, que suele esclerosarse y sus paredes celulares reaccionan dando color azul verdoso o verde intenso con TBO, y una porción distal unistratificada, desflecada y con prolongaciones dentadas o piliformes, formada por células poligonales, cuya reacción de color intensamente púrpura ante el TBO sugiere que están formadas por celulosa o celulosa y cutina.

\section{Clave para determinar las especies arborescentes de Blechnum}

1. Pinnas lineares con láminas completamente enrolladas hacia la cara abaxial; frondas monomorfas de contorno oblan-

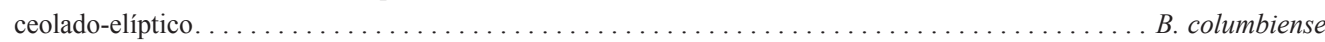

1. Pinnas con láminas planas o sólo revolutas en los márgenes, ni lineares ni enrolladas completamente hacia la cara abaxial; frondas monomorfas o dimorfas de contorno variado $\ldots \ldots \ldots \ldots \ldots \ldots \ldots \ldots \ldots \ldots \ldots \ldots \ldots$

2. Frondas monomorfas oblanceolado-espatuladas, con bases gradualmente reducidas en numerosos pares de pinnas; pinnas adnatas con márgenes planos regularmente aserrados en toda su extensión . . . . . . . . . B. brasiliense

2. Frondas dimorfas de contorno variable, con bases atenuadas o truncadas; pinnas subpecioluladas, sésiles o adnatas; márgenes planos o revolutos, repandos o finamente aserrados sólo en el ápice $\ldots \ldots \ldots \ldots \ldots \ldots \ldots \ldots$

3. Láminas con bases truncadas; pinnas distanciadas, subpecioluladas y márgenes repandos o finamente aserrados sólo

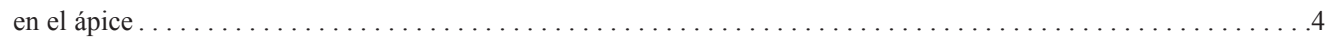

3. Láminas con bases redondeadas o atenuadas y prolongadas en pinnas reducidas, aflébicas o aguijones esclerosados; pinnas próximas, sésiles o adnatas, con márgenes planos y enteros, revolutos en la base o desde la base hasta el

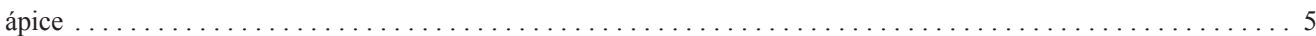

4. Láminas oblongas, pinnas ascendentes, con ápices curvados acroscópicamente, caudados y finamente aserrados

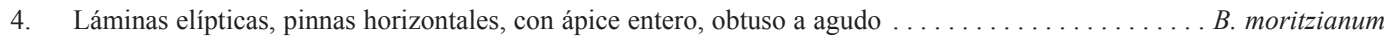

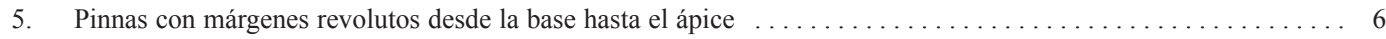

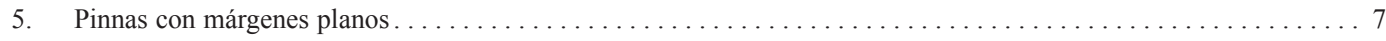

6. Láminas con base redondeada; pinnas sésiles marcadamente ascendentes, linear lanceoladas, con la base atenuada y el ápice agudo u obtuso; estípite con aguijones esclerosados ocasionales . . . . . . . . . . . . B. schomburgkii

6. Láminas con base atenuada y prolongada en pinnas gradualmente reducidas; pinnas sésiles ascendentes, casi tan anchas en la base como en la parte media, con el ápice agudo rígido; estípite siempre con aguijones esclerosados

7. Lámina oblanceolado-elíptica, con ápice agudo y base interrumpida abruptamente o algo atenuada, a lo sumo con 2-4 pares de pinnas basales reducidas; pinnas oblongo-triangulares, próximas, algo incurvadas acroscópicamente, con ápice agudo; estípite con aguijones endurecidos frecuentes $\ldots \ldots \ldots \ldots \ldots \ldots \ldots \ldots \ldots \ldots$ magellanicum

7. Lámina elíptica, con ápice obtuso y base gradualmente reducida, con hasta 14 pares de pinnas basales y con aflebias laminadas; pinnas oblongas u oblanceolado-oblongas, horizontales, imbricadas en el ápice, luego distanciadas, con ápices obtusos u obtuso-mucronulados; estípite $\sin$ aguijones endurecidos $\ldots \ldots \ldots \ldots \ldots \ldots \ldots$ tabulare 


\section{TRATAMIENTO TAXONÓMICO DE LAS ESPECIES}

1. Blechnum brasiliense Desv., Mag. Neuesten Entdeck. Gesammten Naturk. Ges. Naturf. Freunde Berlin 5: 330. 1811. Blechnopsis brasiliensis (Desv.) C. Presl, Epimel. Bot.: 115. 1851. PROTÓLOGO: Habitat in Brasilia (n. v.). TIPO: Brasil. Dombey (P-Jussieu 1390!).

Blechnum corcovadense Raddi, Opusc. Sci. 3: 294. 1819. PROTÓLOGO: Brasil, Corcovado, Raddi. TIPO: Brasil: Rio de Janeiro, Corcovado, Raddi (holótipo PI; isótipos B!, FI).

Blechnum nitidum C. Presl in J. Presl \& C. Presl, Delic. prag. 1: 187. 1822. PROTÓLOGO: Hab. in Brasilia ad Rio-Janeiro. TIPO: Brasil: Rio de Janeiro, Pohl? (isótipos B!, GH).

Blechnum nigrosquamatum Gilbert, Bull. Torrey Bot. Club 24: 258. 1897. PROTÓLOGO: Growing in running water, Colapampa. Collected July 4, 1894, 2314. TIPO: Bolivia. Colapampa, July 1894, Bang 2314 (holótipo no localizado; isótipos B!, GH, NY! US 827874!).

Blechnum brasiliense var. angustifolium Sehnem, Pesquisas, Bot. 3: 529. 1959. PROTÓLOGO: Tal vez se trate de Bl. nitidum Pr. que segundo o Index Fil. é considerado identical con Bl. brasiliense Desv., que Tweedie teria coleccionado no RS (?). TIPO: Brasil: Rio grande do Sul, Vacaria, Passo do Socorro, Sehnem 5747 (holótipo PACA?).

Plantas terrestres o palustres, con rizomas erectos robustos, de $60 \mathrm{~cm}$ o más, no estoloníferos, escamosos. Escamas rizomáticas lineares, enteras, erguidas y algo retorcidas, de color castaño oscuro hasta negruzco, generalmente concoloras o con márgenes más claros, de $2-4 \mathrm{~cm}$ de longitud. Frondas monomorfas de hasta $150 \mathrm{~cm}$ de longitud, ascendentes y arqueadas, las juveniles a veces de color rosado a magenta. Estípites rígidos de hasta $16-18 \mathrm{~cm}$ de longitud, con escamas oscuras en la base, similares a las del rizoma pero en general de color castaño más claro y brillantes. Láminas oblanceoladoespatuladas, pinnatífidas, gradualmente reducidas hacia la base y el ápice, herbáceas firmes a cartáceas. Raquis glabro. Costas glabrescentes o sólo escamosas en la porción abaxial basal, adaxialmente sobresalientes y abaxialmente carenadas; haces inmersos en parénquima, no trabados; protuberancias intercelulares pécticas verrucosas presentes en el tejido parenquimático costal. Segmentos laterales 35-50 pares, ascendentes, completamente adnatos con el raquis, surcurrentes-percurrentes, lanceolado triangulares, planos, con el ápice agudo, acuminado u obtuso y margen regularmente aserrado. Venas visibles, próximas, simples y una vez bifurcadas a distancias variables de la costa, con ramas escasamente divergentes, terminadas en hidatodos grandes de contorno elíptico. Indusios repandos-denticulados, con la mitad basal formada por células engrosadas de contorno sinuoso frecuente, la mitad marginal por células engrosadas de contorno poligonal y el margen mismo formado por ondas irregulares pluricelulares rematadas en dentículos cónicos unicelulares.

Distribución geográfica y ecología: Mesoamérica (Guatemala) y Sudamérica (Colombia, Venezuela, Ecuador, Perú, Bolivia, Brasil, Paraguay, Uruguay y Argentina hasta el delta del Paraná). Moran (1995) cita la especie para Mesoamérica (Guatemala) a partir de una colección única. Crece en selvas o en áreas abiertas, selvas en galería y marginales; es palustre, se encuentra preferentemente en áreas ribereñas de ambiente léntico, en bañados y pantanos, desde los 500 hasta los $1500 \mathrm{~m}$ de altitud; ocasionalmente se encuentra como terrestre en suelos muy húmedos. Forma colonias grandes y abundantes; las láminas varían en textura en relación con el ambiente, y son más coriáceas en los elementos palustres.

Ejemplares de referencia seleccionados: ARGENTINA: Corrientes: Ituzaingó, Rincón del Playadito, $5 \mathrm{~km} \mathrm{~N}$ de San Alonso, islote de selva con arroyo afluente del Playadito, Tressens et al. 5042 (LP). Estancia Santa Rita, interior selva marginal, Tressens et al. 3951 (LP). Misiones: San Ignacio, camino al río, a 200m de la casa de Horacio Quiroga, Palacios 
et al. 1295 (LP). Iguazú, sendero Jacatía, ex Ruta 12, 2km del CIES, Vanni et al. 3239 (LP). Iguazú: Cataratas, Prada s. n. (MA). BRASIL: Goiás: Serra Dourada, Ca. $17 \mathrm{~km}$ of Goiás Velho, Ca. 6km OW of Mossamedes, $750 \mathrm{~m}$, along stream at base of East side of Sierra, Anderson 9957 (LP). Paraná: Yacarehý, Dusén s. n. (MA 213329). Rio de Janeiro: Guanabara, Serra Carioca, Caranta N-638 (LP). Tijuca, Floresta de Tijuca, Castellanos 23070 (LP). Rio Grande do Sul: sin loc., Reinerck \& Czermak 164 (BA). Santa Catarina: Pellegrini, camino de Santo Amaro da Imperatriz a Fazenda Jomar, Krapovickas \& Cristóbal 44827 (LP). Municipio Garuvá, Krapovickas et al. 23073 (CTES). GUYANA: Region PotaroSiparuni, Pakaraima Mts., Upper Ireng. $0.5 \mathrm{~km}$ section of Tay-Slay-O Creek, 4-8km upstream of Ireng mouth, Henkel 873 (MA 571361). PARAGUAY: Canindeyú: cerca de Curguatý, junto al arroyo Schiní, FC5887 (MA 477474). Central: Capiatá, Schinini 4157 (BA). URUGUAY: Cerro Largo, Sierra Aceguá, Osten 18588 (BA).

2. Blechnum columbiense Hieron., Hedwigia 47: 244. 1908. PROTÓLOGO: Columbia, crescit in regione páramo dicta Montis Huila, in páramo de Moras (n. 1268). TIPO: Colombia: Huila: Páramo de Moras, Stübel 1268 (holotipo B!).

Blechnum columbiense var. bogotense Hieron., Hedwigia 47: 244.1908. PROTÓLOGO: Columbia: hábitat prope urbem Bogotá (Karsten s. n.), alio loco in regiones urbem Bogotá, alt. 2800m (Lindig 27). TIPO: Colombia: Bogotá: Karsten s. n. (B!); Lindig 27 (síntipos B!; isosíntipo $27 \mathrm{BM} \mathrm{n.} \mathrm{v.).}$

Blechnum auratum subsp. columbiense (Hieron) Tryon \& Stolze. Fieldiana, Botany 32: 67. 1993.

Plantas terrestres, con rizoma erecto de hasta $200 \mathrm{~cm}$ de alto, escamoso. Escamas rizomáticas aciculares a lineares, de erguidas a algo curvadas todas en la misma dirección, de color castaño oscuro, rígidas, lustrosas, de $3-4 \mathrm{~cm}$ de largo. Frondas monomorfas, pinnadas, de hasta
$1 \mathrm{~m}$ de largo. Estípites rígidos, de hasta $25 \mathrm{~cm}$ de largo, adaxialmente surcados, con escamas basales similares a las del rizoma. Láminas oblanceolado-elípticas, abruptamente reducidas hacia la base y con ápice más o menos obtuso. Raquis y costas densamente escamosos, con escamas castañas largamente acintadas, caducas. Costas adaxialmente surcadas, con un surco profundo, abaxialmente carenadas, con el haz vascular trabado por tejido parenquimático esclerosado y fibroso hacia las caras adaxial y abaxial. Pinna terminal conforme, discreta, a veces más corta que las laterales. Pinnas laterales hasta 40 pares, lineares, coriáceas, marcadamente ascendentes, próximas pero no imbricadas, las apicales sésiles, luego subpecioluladas, con ápice obtuso y lámina completamente enrollada hacia la cara abaxial, con escamas caducas acintadas y largas, enroscadas y de aspecto piliforme. Venas inmersas, distantes, simples y bifurcadas una vez con una división en dos ramas claramente divergentes cerca de la costa, algunas geminadas. Láminas fértiles de hasta $50 \mathrm{~cm}$ de largo, pinnas fértiles angostas, lineares, con margen recurvado y cenosoros mediales que cubren por completo la cara abaxial. Indusios eroso-fimbriados, con células basales engrosadas.

Distribución geográfica y ecología: Páramos de Colombia, Ecuador y Perú, desde 2800 hasta $3600 \mathrm{~m}$ o más.

Observaciones: Tryon \& Stolze (1993) combinaron Blechnum columbiense como una subespecie de $B$. auratum, ésta basada en Lomaria aurata Fée. Blechnum columbiense es una especie bien caracterizada cuyo material tipo no genera dudas sobre su determinación. Se distingue por sus pinnas lineares, angostas, completamente revolutas y enrolladas hacia la cara abaxial, llegando hasta la costa y la ausencia de dimorfismo, ya que las frondas fértiles son indistinguibles de las estériles y sólo en el caso de la presencia de abundantes esporangios maduros se hace visible el carácter de láminas esporógenas. Es afín con B. schomburgkii. Las formas con pinnas más angostas de esta 
última especie, que podrían confundirse con $B$. columbiense, se distinguen por el menor enrollamiento de la lámina, la venación y el número de venas por $\mathrm{cm}$ y por los modelos epidérmicos de epifilos e hipofilos. Blechnum columbiense tiene una distribución geográfica más restringida, limitada a los páramos de Colombia, Ecuador y Perú.

\section{Ejemplares de referencia selecciona-} dos: COLOMBIA: Cundinamarca: Bogotá: Andes, Cordillera Oriental, La Peña, 2 800m, Cuatrecasas 2081 (MA 213330) -2 ejemplares-. Páramo de Montserrate, El Granizo, 3 250m, Fernández Alonso \& Arenas 5134 (MA 517122), planta de la derecha. Páramo de Montserrate, 3 200-3 300m, Cardiel \& Rubi 5 (MA 494869). Carretera Zipaquirá-Paho, 3 000m, Hagemann 165 (COL 118540). Boyacá: Páramo de Guantiva, entre Santa Rosita y Onzaga, en la Quebrada de Sietecueral, 3 410m, Jaramillo Mejía \& van der Hammen 4287 (MA 381471). Entre Zipaquirá y Pacho, Páramo de Guerrero, $3100 \mathrm{~m}$, Murillo \& Villarreal 1370 (MA 228368). Santander: Páramo de Guantiva, lado occidental, hacia Onzaga, 3 460m, Jaramillo Mejía \& van der Hammen 4313 (MA 380907). Cerca Páramo de Santurbán, $3000 \mathrm{~m}$, Barklay \& Araque 185176 (COL 495862). Valle del Río Susa, en el camino de Hato Viejo a La Figuera o Jiquera (Jequera en el mapa), arriba del sitio de Santa Bárbara, en la Loma del Reventón, 3 600m, Jaramillo Mejía \& van der Hammen 4399 (MA 380819). Santander del Norte: al oriente de Pamplona, Páramo de Fontibón, 2 800m, Murillo \& Jaramillo Mejía 1307 (MA 228367). Along road between Pamplona and Chorro Colorado, Vía Chiquirá, 2 580m, Croat 56408 (COL 344728). Plantae Expeditionis Botanicae Mutisii ViceRegni Novae Granatae (1783-1808), 3119 (MA 659308), 3116 (MA 659331), 3123 (MA 659334). ECUADOR: Loja: Cajanuma, Lehnert 833 (UC 1791891). PERU: Luya: Distrito Camporredondo, Tullanya, cerro Wiscocunga, Campos \& Campos 3138 (UC 1733388). Oxapampa: Huancabamba, Sector Santa BárbaraMilpo, 2 750-3 000m Mellado \& Ortiz 2745
(UC 1870542). Piura: Huancabamba, Hutchinson 1619 (UC, USM).

3. Blechnum cycadifolium (Colla) J.W. Sturm., Abh. naturhist. Ges. Nürnberg 2: 173. 1858. Lomaria cycadifolia Colla, Herb. pedem. 6: 219. 1836. Blechnum magellanicum var. cycadifolium (Colla) C. Chr., Dansk Bot. Ark. 10(2): 9. 1910. Struthiopteris cycadifolia (Colla) Ching, Sunyatsenia 5: 243. 1940. PROTÓLOGO: [Lomaria] magellanica an diversa? Berter. In sylvaticis collium et montium ins. Juan-Fernández. TIPO: Masatierra, Yunque. 24.08.1908, Leg.: C. J. F. Skottsberg 407. Notes: Expeditio suecica 1907-1909. (Holótipo, isótipo B!).

Lomaria lanuginosa Kunze, Analecta pteridogr.: 19. 1837. Blechnum lanuginosum (Kunze) J.W. Sturm, Abh. naturhist. Ges. Nürnberg 2: 175. 1858. PROTÓLOGO: Ex insula Juan Fernandez misit Larrain, Decembri 1829 lectam (Lomariae sp. n.? $\mathrm{n}^{\mathrm{o}}$ 1110). Fertilem dedit Bory de St.Vincent; sterilem unio itinerar. e plant. Berteroanis (Herb. propr.).

Plantas terrestres, de hasta $300 \mathrm{~cm}$ de alto, con rizoma erecto escamoso de hasta $250 \mathrm{~cm}$. Escamas rizomáticas lineares, erguidas a algo curvadas todas en la misma dirección, de color castaño oscuro, rígidas, de $3-6 \mathrm{~cm}$ de largo. Frondas dimorfas, pinnadas, de hasta $120 \mathrm{~cm}$ de largo, las estériles más largas que las fértiles, éstas erguidas, de hasta $80 \mathrm{~cm}$ de largo. Estípites rígidos, de hasta $30 \mathrm{~cm}$ de largo, adaxialmente surcados y con pinnas vestigiales esclerosadas reducidas a aguijones espinosos. Láminas estériles oblanceoladas, reducidas hacia la base y con ápice obtuso a mucronado. Raquis y costas con escamas castañas, caducas. Costas adaxialmente surcadas con un surco profundo y abaxialmente carenadas, con un solo haz vascular trabado por tejido esclerosado y fibroso que se extiende hacia las caras adaxial y abaxial. Pinna terminal conforme o más corta que las laterales, a veces adnata con 1-2 pares apicales. Pinnas laterales hasta 50 pares, coriáceas, ascendentes, imbricadas en el tercio apical y luego muy próximas, sésiles, de 
contorno elíptico angosto, ápice obtuso rígido en plantas adultas, margen repando revoluto, abaxialmente escamosas en la costa, con escamas caducas. Venas inmersas, distantes, simples y bifurcadas una vez cerca de la costa en dos ramas claramente divergentes, algunas geminadas. Láminas fértiles erectas, erguidas en el centro de la espiral de frondas; pinnas fértiles angostas, lineares, con cenosoros mediales a marginales. Indusios eroso-fimbriados, con células basales engrosadas.

\section{Distribución geográfica y ecología:} Chile, Juan Fernández. En general, se la considera como una especie endémica de las islas Robinson Crusoe y Alejandro Selkirk, donde convive con B. magellanicum. En la isla Robinson Crusoe forma colonias abundantes que se distribuyen desde el Sector de Puerto Francés hasta Cerro Chumacera; en Cordón y Quebrada de Salsipuedes, Cerro Alto, Sector de Puerto Inglés hasta Quebradas Vaquería y Juanango; en isla Alejandro Selkirk se encuentran en Quebrada las Chozas, Quebrada de las Casas, Quebrada la Lágrima, Quebradas los Inocentes, El Guatón, La Lobería, Varadero, Óvalo, el Pasto, El Sándalo, Planos de Sánchez (Johow 1896, Skottsberg 1924, 1953, Barrera 1997). Rodríguez Ríos (1995) y Ricci (1990, 1992) registraron más de 5000 plantas distribuidas en diferentes sectores de ambas islas, donde la especie es abundante en lugares abiertos y forma agrupaciones muy tupidas. Esos autores hacen referencia a la regeneración natural de las colonias pero también consideran que a pesar del gran número de plantas que se detectaron en ambas islas, la especie estaría amenazada por el progreso de Rubus ulmifolius Schott (zarzamora), Aristotelia chilensis (Molina) Stuntz (maqui) y Ugni molinae Turcz. (murtilla), así como por los incendios y los deslizamientos de terreno producidos por las frecuentes lluvias. Ricci $(1990,1992)$ la considera fuera de peligro, mientras que para Rodríguez Ríos (1995), Baeza et al. (1998) y Danton (2004) sería vulnerable.
Observaciones: Blechnum cycadifolium forma comunidades muy densas en lugares abiertos o despejados, hasta los $1200 \mathrm{~m}$ de altitud. Es abundante en matorrales y pastizales, forma extensos bosquecillos, aún en suelos erosionados. Muestra cierta variación en la textura y grado de desarrollo de las frondas de acuerdo con la cantidad de luz que recibe y se han mencionado formas umbrófilas (Johow 1896). Según Skottsberg (1953), es más abundante en las faldas expuestas al viento, en Masatierra, donde es la planta dominante y crece desde el nivel del mar hasta las cumbres más elevadas. En Masafuera es menos frecuente y asciende por encima del límite arbóreo hasta cerca de la cima de la isla. Es una de las especies arborescentes más robustas del género, ya que su rizoma erecto puede alcanzar los $250 \mathrm{~cm}$ de altura. Las colecciones son escasas.

Ejemplares de referencia seleccionados: CHILE: Archipiélago de Juan Fernández: Masafuera, Correspondencia Camp, Skottsberg \& Skottsberg 405 (US 1085782). Los Inocentes, Solbrig et al. 3760 (US 2421520). Masatierra: high ridge above Rabanal, Skottsberg \& Skottsberg 580 (US1055780). Masatierra, Aréte del Centinela, Skottsberg \& Skottsberg 580 (P). Slopes of Damajuana, Solbrig et al. 3790 (US 2421535). Ex insula Juan Fernández, Bertero s. $n$. (P 0140440).

4. Blechnum magellanicum (Desv.) Mett. Fil. Lechl. 1. 14. 1856. Lomaria magellanica Desv. Mag. Neuesten Entdeck. Gesammten Naturk. Ges. Naturf. Freunde Berlin 5: 330. 1811. PROTÓLOGO: [¿Chile?] Habita ad fretum magellanicum $\mathrm{n}$. $\mathrm{v}$.

Plantas terrestres con rizoma erecto de hasta $100 \mathrm{~cm}$ de alto y $20 \mathrm{~cm}$ de diámetro, cubierto por las bases persistentes de los estípites caducos, escamosos. Escamas rizomáticas lineares, erguidas a algo curvadas todas en la misma dirección, enteras, largamente atenuadas, sésiles, de $3.5-4 \mathrm{~cm}$ de largo. Frondas dimorfas; las estériles arqueadas, de $50-150 \mathrm{~cm}$, las fértiles erectas, iguales o algo más largas. Estípites rígidos, 1/3 del largo total de la 
fronda, paleáceos, con un surco adaxial, con aguijones o mamelones esclerosados y sección casi circular; internamente huecos, con área central parenquimática reabsorbida. Lámina estéril oblanceolado-elíptica, ancha, con ápice agudo y base interrumpida abruptamente y sin pinnas reducidas ni aflebias laminares en frondas adultas, en éstas con aguijones esclerosados resultantes de la atrofia de pinnas y algo atenuada, con 2-4 pares de pinnas reducidas no esclerosadas en frondas jóvenes. Raquis paleáceo. Costas acanaladas en la cara adaxial y casi carenadas en la abaxial, externamente con glándulas unicelulares de mucílago y escamas lineares acintadas con aspecto lanuginoso, con el haz vascular trabado por tejido parenquimático esclerosado, fibroso, hacia las caras adaxial y abaxial. Pinna terminal conforme con las laterales pero en general más angosta, más ancha en la base y adnata con uno o más pares. Pinnas laterales hasta 40 pares, coriáceas, alternas o sub-opuestas, oblongo-triangulares, con ápices agudos atenuados y bases adnatas en el tercio apical de las frondas, luego sésiles y truncadas, asimétricas, con margen repando plano. Láminas fértiles erectas, con pinnas de $0.5 \mathrm{~cm}$ de ancho, dirigidas acroscópicamente. Indusio con margen irregular.

Distribución geográfica y ecología: Argentina y Chile. En Argentina desde Neuquén hacia el $\mathrm{S}$, en bosques hiperhúmedos cordilleranos y en áreas más expuestas, sobre todo en los bosques subantárticos, en suelos sueltos o algo rocosos, no acidófila; en Chile, desde Valparaíso y el Archipiélago de Juan Fernández hacia el S, en bosques, bordes de bosques, valles y quebradas húmedas, entre rocas y en tierra, con agua cerca pero no en suelos encharcados. En el archipiélago de Juan Fernández en asociaciones con B. cycadifolium y B. schottii (Colla) C. Chr., un elemento de las áreas más húmedas, escandente o hemiepífito.

Ejemplares de referencia seleccionados: ARGENTINA: Chubut: Lago Menéndez, Alerzal del Norte, Meyer 9517 (LIL). Islas Malvinas: Puerto Stanley, Hicken 31 (LIL). Neuquén:
Isla Victoria. Nahuel Huapi, Diem 1914 (LIL). Río Negro: Laguna Frías, Meyer 7338 (LIL 113135). Santa Cruz: Lago Argentino, Brazo Mayo, Vervoorst 4806 (LIL). Tierra del Fuego: Isla de Los Estados, Puerto Cook, below the multiple peaks of Monte Orejas de Burro, Dudley \& al. 692 (LP). Tierra del Fuego: Torres 1179 (LP). CHILE: Región V: Valparaíso, Archipiélago de Juan Fernández: Isla Robinson Crusoe (Mas a Tierra), Pérez Moreau 22936 (BA). Plazoleta del Yunque [Plaz. del Yunque, im Hochwalde on volcán, 300m], Bock 33 (US 15994193 \& US 1787893). Mountain apur E of Quebrada de la Damajuana, Skottsberg \& Skottsberg 74 (US 2751260). Region XII: Provincia Última Esperanza, Puerto Edén, Isla Wellington, Capitanía de Puerto, Pisano 6455 (CONC 104825, MA 700919). Fiordo Peel, Río Martillar, Pisano 5942 (CONC 95870, MA 7000121). Bahía Micaela, Isla Saumarez, canal Grappler, Pisano 6573 (CONC 104778, MA 700330). [Chile ] Pto. Egmont, Née s. n. (MA 230549).

5. Blechnum moritzianum (Klotzsch) Moore. Ind. fil.: 200. 1857. Lomaria moritziana Klotzsch, Linnaea 20: 347. 1847. TIPO: Venezuela: Mérida, in sylv. subalpin. J. W. K. Moritz 301 (B 20 0031858!, B 200031859 !, B 20 0031860!, B 200031861!1; isótipos $\mathrm{K}$ !, BM!).

Blechnum yungense Ramos Giacosa, Novon 20(1): 68-72. 2010. TIPO: Argentina: Jujuy: Depto. Ledesma, camino a Valle Grande, 9.IV-1971, Legname \& Cuezzo 8219 (Holótipo LP!, Isótipos LIL!, CTES!).

Plantas terrestres, con rizomas ascendentes a erguidos, de hasta $70-80 \mathrm{~cm}$ de alto, escamosos. Escamas rizomáticas lineares, curvadas todas en la misma dirección, rígidas, de color castaño oscuro, concoloras o bicoloras y con un área central castaño-negruzca, sésiles, enteras, largamente atenuadas, de $2-3 \mathrm{~cm}$ de largo. Frondas dimorfas, las estériles de hasta $150 \mathrm{~cm}$ de largo, con láminas elípticas, anchas, con ápices agudos, bases truncadas sin aflebias ni pinnas reducidas; las fértiles iguales o más 
largas. Estípites de hasta $30-35 \mathrm{~cm}$ de largo o más a veces tan largos como la lámina, adaxialmente surcados, con escamas basales similares a las rizomáticas. Raquis escamosos, con escamas largas y lineares, castañas. Costas abaxialmente curvas y adaxialmente surcadas, con un surco poco profundo, con el haz trabado hacia la cara adaxial por tejido parenquimático esclerosado que también forma casquetes fibrosos hipodérmicos hacia la cara abaxial, que no siempre traban el haz. Láminas estériles pinnadas con la pinna terminal conforme, algo más corta y basalmente más ancha que las laterales. Pinnas laterales hasta 25-30 pares, lanceoladas a oblongo-lanceoladas, horizontales, más o menos distanciadas, con bases asimétricas cuneadas, ápices agudos poco atenuados y márgenes repandos planos. Venas inmersas próximas, simples y una vez bifurcadas cerca o lejos de la costa, geminadas raras o ausentes. Indusios profundamente eroso-desflecados.

Distribución geográfica y ecología: Sudamérica, desde Venezuela hasta el NO de Argentina. Es una especie abundante en los Andes; crece tanto en la selva montana como en el bosque parcialmente caducifolio más alto, en pastizales y pajonales, sobre barrancas y laderas expuestas. Es una planta terrestre, resistente y rígida, de color verde más claro a glauco en los hipofilos. Desde los 1 200-1 330m hasta los $3500 \mathrm{~m}$ de altitud.

Observaciones: Lomaria moritziana Klotzsch fue transferida a Blechnum por Moore (1857), quien incluyó en su sinonimia Salpichlaena volubilis (Kaulf.) Sm. Esta combinación es válida de acuerdo con el ICBN, y Moore (1857), además, incluye una breve referencia al basónimo, acompañada del nombre de su autor: "Moritzianum, Kl.". Hieronymus (1908) aceptó la combinación de Moore (1857) pero excluyó, correctamente, la sinonimia, citó tanto el basónimo como la nueva combinación en sus comentarios sobre las afinidades de $B$. guascense Hieron. y también citó el tipo, procedente de Venezuela (Moritz 301), depositado en el Herbario B. Es el último autor que utilizó ese nombre, ya que no se encuentra en obras posteriores y B. moritzianum aparece en los herbarios determinada indistintamente como B. auratum subsp. auratum, B. schomburgkii o B. buchtienii. Blechnum moritzianum es el nombre que corresponde aplicar a los materiales recientemente descritos por Ramos Giacosa (2010) como B. yungense. El tipo depositado en $\mathrm{B}$ es un fragmento de fronda y son más representativos los isótipos depositados en BM y $\mathrm{K}$, en los que las frondas están completas e incluyen estípites con sus escamas basales curvadas. Blechnum moritzianum difiere de $B$. schomburgkii por el contorno de las láminas, las pinnas horizontales anchas y distanciadas, de láminas bien desarrolladas, con bases asimétricas subpecioluladas y márgenes enteros planos, no revolutos. La especie más afín es $B$. werckleanum, con pinnas patentes incurvadas, oblongas angostas, atenuadas, caudadas, con ápices acuminados aserrados dirigidos acroscópicamente, marcadamente bicolores y abaxialmente mucho más claras, un rasgo que también puede estar presente en $B$. moritzianum pero es más evidente aún en material seco, en $B$. werckleanum. Los modelos epidérmicos de las tres especies difieren en la ondulación de los epifilos, más somera en $B$. schomburgkii y B. werckleanum, y de los hipofilos, con ondas simples, subangulosas en $B$. schomburgkii, curvas, en $B$. werckleanum y compuestas curvas en $B$. moritzianum. Las hipodermis también difieren, ya que en $B$. schomburgkii está formada por células largas traqueiformes, lignificadas mientras que en $B$. moritzianum y $B$. werckleanum las células son más cortas, menos engrosadas y no se esclerosan.

Ejemplares de referencia seleccionados: ARGENTINA: Salta: Parque Nacional Baritú, Los Toldos, camino al Lipeo, Prada s. n. (LP, MA). Santa Victoria, entre El Lipeo y Canto del Monte, 200m, Novara \& Adzet 9133 (CORD, CTES, MCNS). Camino Los Toldos al Lipeo, Desvío San José, 1750m, Cassá et al. 286 y 287 (LP, MCNS). Canto del Monte, camino de Los Toldos, $6 \mathrm{~km}$ al $\mathrm{N}$ antes de llegar a Lipeo, 1200-1500m, Novara \& Barboza 11556 (CTES, 
MCNS). Santa Victoria, camino al Lipeo, $1600 \mathrm{~m}$, Martinez \& de la Sota s.n. (MCNS). BOLIVIA: Santa Cruz: Florida, Parque Nacional Amboró, 8km (by air) NE of Mairana, along trail and near ridge tops, $5.5 \mathrm{~km} \mathrm{~N}$ of Campamento La Yunga Park Station, 2300m, Sundue et al. 545 (UC 1780949). Caballero, Cerro Curucucho, ca. $1 \mathrm{~km}$ ENE de Liberia, $3000 \mathrm{~m}$, Saldias \& Fernández 4564 (CTES 291019). COLOMBIA: Chocó: Municipio San José del Palmar, Cerro del Torrá, Silverstone-Stopkins et al. 1374 (CTES). Bogotá: montes detrás de Bogotá, 3200m, Carvajalino 214 (COL 89678). Boyacá: Sierra Nevada de Cocuy, Cordillera Oriental, Grubb \& Guymer 66 (COL 89414). Cundinamarca: San Miguel, extremo SW de la Sabana de Bogotá, $2800 \mathrm{~m}$, Jaramillo Mejía, Murillo \& van der Hammen 3418 (MA 381510 y MA 380762). Below Alto del Cuchuco, $7 \mathrm{~km}$ SW of Sibate, Tryon \& Tryon 6111 (COL 95378). Zipaquirá, inmedianciones de Pantano Redondo, 2900m, Huertas \& Camargo 580 (COL 106268). Al N de Bogotá, bosques arriba de la Hacienda La Francia, Torquita, 2800m, Jaramillo Mejía \& van der Hammen 4522 (COL 243992). Meta, Cordillera Oriental main north affluent of Rio Grande, south of Cordillera de Las Cruces, south slopes of Páramo de Sumapaz, 3130m, Fosberg 20866 (COL 244351). Santander del Norte: al oriente de Pamplona, Páramo de Fontibón, 2800m, Murillo \& Jaramillo Mejía 1321 (COL 125892). ECUADOR: ZamoraChinchipe: Estación Científica San Francisco, Lehnert 849 (UC 1791755). PERÚ: Amazonas: Chachapoyas, Chachapoyas-Cajamarca road, between Limebamba and Calla-Calla, 12-15km from Leimebamba, coll? 4984 (USM 214613). Pasco: Oxapampa, from Antenna past Chacos to the Laguna, van der Werff et al. 19642 (UC 1872875). Piura: Ayabaca, Bosque de Huamba, Cano 1613 (USM).

\section{Blechnum schomburgkii (Klotzsch)} C. Chr., Index filic.: 159. 1905. Lomaria schomburgkii Klotzsch, Linnaea 20: 346. 1847. PROTÓLOGO Y TIPO: Guiana angl. Richard Schomburgk n. 1162 (holotipo B!, fragm $\mathrm{NY}$ !, isótipo $\mathrm{K}$ ).
Blechnum schomburgkii (Klotzsch) C.Chr. var. squamulosa Hieron., Plantae Stübelianae, Filices, no. 444. TIPO: Colombia: Bogotá Excurs.; oberhalb Pasca. Alt.: 9000'. A. Stübel 444 (B 200033242 !).

Lomaria aurata Fée, Mém. foug. 8: 71. 1854-57. Blechnum auratum (Fée) R. M. Tryon \& Stolze subps. auratum, Fieldiana, Bot. 32: 67. 1993. PROTÓLOGO: Nouvelle-Grenade, province d'Ocaña, dans les Paramos, à 2600-3300 mètres d'altitude (L. Schlim ${ }^{\circ}$ 394; 1846-1852). TIPO: Colombia: Ocaña, Schlim 394 (holotipo P!).

Blechnum buchtienii Rosenst., Repert. Spec. Nov. Regni Veg. 5: 231. 1908. Struthiopteris buchtienii (Rosenst.) Maxon \& C. V. Morton, Bull. Torrey Bot. Club 66: 44. 1939. PROTÓlOGO: Yungas borealis; Unduavi, 3800m alt., in silvis, 12-II-1907., Dr. O. Buchtien $\mathrm{n}^{\circ}$ 878. TIPO: Bolivia (Nor Yungas), Unduavi, Buchtien 878 (holotipo B!, isótipo US 867062!).

Lomaria obtusifolia Presl, Tent. Pterid. 143. 1836. Blechnum obtusifolium (Ettingsh.) C. Chr., Denkschr. Kaiserl. Akad. Wiss. Wien 23: 59. 1864. TIPO: [Según Presl]"Herb. Bras. Reg. Berol. 101". Según Dittrich (2005), hay dos ejemplares con este nombre: Brasilia, Sellow.1825 (B 20-0031991!) y otro en BM, con la misma indicación.

Lonchitis-aspera tabularis var. saligna Farwell, Amer. Midl. Naturalist 12: 277. 1931. TIPO: Bolivia, Mapiri, Rusby 313 (holotipo? isótipo US 834067!).

Plantas terrestres, de hasta $3 \mathrm{~m}$ de altura, con rizomas erectos no estoloníferos, escamosos. Escamas rizomáticas aciculares rígidas, curvadas todas en la misma dirección, pluriestratificadas, con área central oscura y márgenes hialinos, lustrosas, de $2.5-4.5 \mathrm{~cm}$ de largo. Frondas dimorfas, con láminas oblanceoladoelípticas, pinnadas, coriáceas. Estípites rígidos, con un surco adaxial y escamas basales similares a las del rizoma. Raquis y costas con escamas triangulares largas, pardo-amarillentas, concoloras o discoloras, caducas a persistentes. Costas abaxialmente carenadas y adaxialmente 
surcadas, con un surco profundo, con el haz vascular trabado por tejido parenquimático esclerosado y fibroso hacia las caras adaxial y abaxial. Pinna terminal discreta, linear o linear lanceolada, conforme pero más corta y angosta que las laterales, con ápice agudo rígido y base uni o bi-auriculada, a veces adnata con el primer par apical. Pinnas laterales hasta 40-50 pares, sésiles, marcadamente ascendentes, próximas pero no imbricadas, lanceoladas angostas a anchas, discretas, con ápices agudos u obtusos, márgenes repandos revolutos desde la base hasta el ápice, carenadas, con venas distanciadas inmersas pero visibles, simples y bifurcadas una vez cerca de la costa, algunas geminadas, con una sola dicotomía de ramas claramente divergentes. Indusio cartáceo, firme, de color castaño claro, submarginal o marginal, lacerado, de hasta $0.5 \mathrm{~cm}$ de ancho.

Distribución geográfica y ecología: Mesoamérica, Sudamérica (Colombia, Venezuela, Ecuador, Perú, Bolivia, Brasil, Paraguay). Crece en selvas montanas y pedemontanas, en bordes de selvas, áreas boscosas húmedas, hasta pantanosas y desde los 1400-1500 hasta los $3800 \mathrm{~m}$ de altitud.

Observaciones: Blechnum schomburgkii es una especie insuficientemente caracterizada hasta el momento. Tryon \& Stolze (1993) la consideran diferente de B. auratum subsp. auratum y tratan sub $B$. schomburgkii las formas con pinnas patentes, anchas y con márgenes planos que aquí se han agrupado sub $B$. moritizianum o $B$. werckleanum, ambas especies abundantes en Perú. Moran (1995) no menciona $B$. auratum subsp. auratum y trata el material mesoamericano como $B$. buchtienii. Dittrich (2005) señala que es una especie variable, posiblemente afín con B. tabulare, señalando que requiere más estudios. Ramos Giacosa (2010) describe B. yunguense sobre material argentino de $B$. moritzianum y señala que las diferencias con B. schomburgkii se vinculan con el contorno de la lámina, la anchura de las pinnas, las escamas y las esporas. El material tipo de B. schomburgkii depositado en el herbario B, incluyendo el de B.schomburgkii var. squamulosa, ilustran la variación morfológica de la especie, cuyas pinnas son ascendentes, de contorno linear a lanceolado angosto con ápices agudos a obtusos, bases sésiles y márgenes revolutos. El tipo de Lomaria aurata Fée, basónimo de $B$. auratum subps. auratum, que se encuentra en el herbario $\mathrm{P}$ y el de $B$. butchienii son considerados aquí también como parte de la variación morfológica esperable de la especie. Las pinnas de B. schomburgkii varían en rigidez y enrollamiento marginal en ejemplares juveniles y adultos, pero las láminas no alcanzan el desarrollo que se observa en $B$. moritzianum o en $B$. werckleanum. La mencionada variación externa de $B$. schomburgkii ha derivado en determinaciones diferentes, ya que en los herbarios aparece indistintamente como $B$. auratum subsp. auratum, B. buchtienii o $B$. schomburgkii. Del mismo origen puede ser la determinación de ejemplares de $B$. moritzianum como B. schomburgkii. Dittrich (2005) lo reconoce para Brasil, señala que tiene afinidades con $B$. tabulare, e incluye en la sinonimia a B. exiguum Dutra y B. bradei Markgraf, cuyos tipos proceden de Rio Grande do Sul. El holótipo R de B. exiguum no ha sido visto aquí, aunque sí se ha podido consultar el isótipo ICN. El tipo de $B$. bradei en B parece muy similar al isótipo ICN de $B$. exiguum, los nombres podrían ser sinónimos y ambos materiales están dentro de la variación de $B$. tabulare más que de B. schomburgkii. La distribución geográfica de $B$. schombugkii parece más bien mesoamericana y sudamericana andina y tal vez la especie no se encuentre realmente en Brasil, un área en la que en cambio, está presente $B$. tabulare en ambientes que no serían los típicos de B. schomburgkii, una planta de pajonales de altura, selvas, bosques montanos y páramos.

Ejemplares de referencia seleccionados: BOLIVIA: Cochabamba: Chapare, Caja-Región, Llanta Aduana, Tarija, 3200m, Steinbach 9551 (LIL 20657). Chapare, Incachaca-San Antonioni, 2600m, Steinbach 9062 (LIL 96885). Santa Cruz, Ruinas Incaicas-El Fuerte, 2000m, Steinbach 8259 (LIL 20684) 
-planta de de la izquierda, la de la derecha es $B$. tabulare. COLOMBIA: Cundinamarca: PNN, Chingaza, Sendero Suasie, Acosta 024 (COL 466668). Al N de Bogotá, bosque arriba de la Hacienda La Francia (Torquita), 3 030m, Jaramillo Mejía, Murillo \& van der Hammen 4575 (MA 380245). Tolima: Cordillera Oriental, vertiente oriental, bajando del Ruiz, carretera entre Letras y balcones, 3400-3200m, Cuatrecasas 9365 (COL 35766). COSTA RICA: Limón: Cordillera de Talamanca, Atlantic slope, unnamed cordillera, between Rio Terbi and Rio Siní, Davidse et al. 28996 (UC 1506870). San José: Villa Mills, hacia el Cerro de la Muerte, Gabriel y Galán \& Sundue s.n. (MA). ECUADOR: Azuay: Sevilla de Oro, en el camino de Sevilla de Oro-Méndez, llegando al Páramo del Castillo, Garmendia \& Cisneros 1138 \& 1106 (MA 630212 \& 630213). Carchi, Tulcán, Camino Tufio, Aguas Hediondas en la base del Volcán Chiles, límite con Colombia, 3 650m, Palacios 11823 (UC 1744624). Loja: km 51 on Panamerican Highway, 2900m, Holm-Nielssen et al. 4677 (UC 1477913). PERÚ: Cuzco: Camino a Buenos Aires, Kcosñipata, Peralta 26 (USM 214611). Mariscal Cáceres, Río Abiseo National Park, 3300m, s. coll. 2762 (USM 83386). Puno, Sandía, 3250-3400m, Metcalf 30519 (LIL 146372). San Martín: Mariscal Cáceres, Chochos, NW corner of Río Abiseo National Park, 3500m, Young \& León 4824 (USM 214612). VENEZUELA: Amazonas: Depto Atabapo, Cerro Marahuaca, Liebner 24709 (UC 1557150). Trujillo: Municipio Boconó, Parque Nacional Guaramacal, Páramo de Vicuyal, 2730m, Stergios 20148 (UC 1784887).

7. Blechnum tabulare (Thunb.) Kuhn, Fil. Afr.: 94 (1868). Pteris tabularis Thunb., Prodr. pl. cap.: 171 (1800). Lomaria tabularis (Thunb.) Mett. ex Baker, In Mart., Fl. bras. 1(2): 418 (1870). TIPO: [Sudáfrica] e Cap b. Spei, in lateribus (aquo) sis summi Taffelberg, Thunberg s.n. (Holótipo UPS-THUNB 24965!).

Lomaria imperialis Fée \& Glaziou in Fée, Cryp. vasc. Brésil 1: 21. 1869. Blechnum imperiale (Fée \& Glaz.) H. Christ, in Schwacke, Pl. nov. mineir. 2: 27. 1900. Struthiopteris imperialis (Fée \& Glaziou) Ching, Sunyatsenia 5: 243. 1940. TIPO: Brasil: Río de Janeiro, Serra dos Órgãos, Glaziou 2801 (holotipo P!, isótipo $\mathrm{B}$ !, foto $\mathrm{BM}$ !).

Blechnum spannagelii Rosenst., Hedwigia 46: 93. 1907. PROTÓLOGO: [Brasil, Estado Santa Catharina] Lages, an schattigen Orten, C. Spannagel n. 86. TIPO: Brasil: Santa Catarina, Lages, Spannagel 86.1 (holótipo B!; isótipos HB, NY!, S, UC!, US!).

Blechnum spannagelii f. pectinata Rosenst., Hedwigia 46: 93. 1907. PROTÓLOGO: [Brasil, Estado Santa Catharina] Lages, C. Spannagel n. 86.1. TIPO: Brasil: Santa Catarina, Lages, Spannagel 86.1 (holótipo B!; isótipos $\mathrm{HB}, \mathrm{NY}$ !, $\mathrm{S}, \mathrm{UC}$ !, US!).

Blechnum madagascariense Tardieu, Mém. Inst. Sci. Madagascar, Sér. B, Biol. Vég. 6: 230, f. 3. 1955. PROTÓlOGO Y TIPO: Madagascar: Vallée de la Lokoho (NE), Mont Beondroka au nord de Maroambihy, rochers de la crête, 1400-1450m, Humbert H. 23466 (Holótipo?, Isótipos P00518459! P00483203!).

Plantas terrestres, con rizoma erecto de hasta $120 \mathrm{~cm}$, cubierto por las bases persistentes de los estípites, escamoso. Escamas rizomáticas concoloras o discoloras y con un área central castaño-negruzca, sésiles, lineares, enteras, largamente atenuadas y terminadas en una sola célula, laxas o rígidas, curvadas todas en la misma dirección, con frecuencia retorcidas helicoidalmente, de hasta $6 \mathrm{~cm}$ de largo. Frondas agrupadas erectas y rígidas o algo divergentes y laxas, las estériles de hasta $200 \mathrm{~cm}$ de largo y las fértiles más largas. Estípites de $10-25 \mathrm{~cm}$ de largo (los fértiles más largos y algo más gruesos), estriados o arrugados, casi glabros excepto en la base que es densamente paleácea, con escamas similares a las del rizoma y con glándulas mucilaginíferas cilíndricas, esparcidas; internamente masivos. Raquis y costas subglabros, con glándulas mucilaginíferas unicelulares dispersas. Costas abaxialmente curvas y adaxialmente surcadas, con un surco poco profundo, internamente con 
el haz inmerso en tejido esclerosado. Lámina estéril de hasta $110 \mathrm{~cm}$, elíptica, con ápice obtuso y base cuneada, gradualmente reducida, con hasta 14 pares de pinnas basales más distanciadas que las apicales, que pueden estar casi imbricadas en el ápice y con aflebias laminadas. Pinna terminal adnata con otros pares apicales, conforme, oblonga, más corta que las laterales. Pinnas laterales 40-42 pares, horizontales a ascendentes, oblongas, distanciadas, variablemente adnatas con el raquis a sésiles, con láminas planas, ápices obtusos a obtusomucronulados y márgenes repandos planos o basalmente revolutos; venas próximas, simples, geminadas y bifurcadas, las bifurcadas divididas una o dos veces a distancias variables de la costa, adaxialmente inmersas y abaxialmente visibles, sin hidatodos o con éstos poco apreciables. Indusio cartáceo, firme, de color castaño claro, submarginal o marginal, lacerado, de hasta $5 \mathrm{~mm}$ de ancho.

Distribución geográfica y ecología: Sudamérica: Argentina, Brasil, Bolivia, Paraguay y Uruguay. África: Nigeria, Camerún, Gabón, Zaire, Ruanda, Burundi, Etiopía, Zambia, Malawi, Mozambique, Zimbabue, Tanzania, Uganda, Congo, Angola, Kenia, Sudáfrica, Islas del océano Índico (Madagascar, Reunión, Mauricio) y del océano Atlántico (Marianas, Tristán da Cunha). En América tropical y subtropical crece en bosques húmedos, bordes de selvas, depresiones húmedas, cerca de áreas anegadas, en pastizales, pajonales y praderas graminosas, formando poblaciones no muy abundantes; las plantas pueden ser acidófilas y asociarse en áreas encharcadas con Sphagnum. Toleran la exposición (no son frecuentes en selvas cerradas) pero requieren agua abundante en el suelo y crecen hasta los $2000 \mathrm{~m}$. En África crece en selvas montanas como terrestre o epipétrica, a lo largo de corrientes de agua, no es edáficamente selectiva y produce frondas fértiles solamente durante el verano (Burrows 1990); se encuentra desde los 900 hasta los $1600 \mathrm{~m}$ de altitud.
Observaciones: Aquí se ha estudiado material adicional, no analizado en estudios previos de B. tabulare (Rolleri et al. 2008), de toda su área de distribución, que incluye América tropical y África. La especie presenta cierta variación en el grado de división de la lámina, desde pinnas sésiles hasta adnatas, condición que es común tanto en ejemplares americanos como africanos; el extremo de esta condición corresponde en parte a plantas previamente asignadas a $B$. madagascariense y $B$. spannagelii, ésta considerada hasta ahora endémica de Brasil, aunque aquí se ha visto material de Paraguay con esa morfología. Blechnum spannagelii fue considerado afín con $B$. brasiliense por Dittrich (2005) por la presencia en ambas de láminas pinnatisectas. Se la ha incluido en $B$. tabulare por los caracteres externos e internos tales como división de las láminas, contorno y márgenes de las pinnas, modelos epidérmicos, estructura de las costas, tipo de hipodermis y mesofilo. En relación con B. madagascariense, no hay menciones ni estudios posteriores a su diagnosis y descripción (Tardieu Blot 1955, 1960), pero esa autora ya sugiere que podría ser una "forma" reducida o pequeña de $B$. tabulare y esa sugerencia se ajusta a lo observado aquí en la morfología vegetativa y en Passarelli et al. (2010), en las esporas, aunque en ese trabajo se ilustraron separadamente de las de B. tabulare, por estar inconcluso el estudio actual. Los caracteres que llevan a incluirla en $B$. tabulare son los mismos señalados para $B$. spannagelii. Blechnum tabulare es común en áreas selváticas del S-SE de Brasil, Paraguay, NE y NO de la Argentina y Uruguay, y se la ha confundido con $B$. schomburgkii, que se considera aquí una especie preferentemente andina, de las laderas orientales de los Andes, páramos y áreas montañosas de Venezuela y las Guayanas, menos común en áreas selváticas. El límite de área de ambas coincide en regiones montanas de las Yungas (Bolivia y NO de la Argentina).

Ejemplares de referencia seleccionados: AFRICA. BURUNDI: Burundi: Nwisare, presso Burundi, Pichi Sermolli 7035 (UC 429945). MADAGASCAR: Antananarivo: Massif 
d'Itremo, W of Ambositra, van der Werff \& McPherson 13587 (UC 1604667). Antsiranana: Montagne d'Ambre PN, near sommet d'Ambre, Andrianantoanina et al. 54 (UC 1872011). R. N. 1, 50km W of Antananarivo, Tapia forest, Barnett et al. 457 (UC 1549512). Antsiranana: Andapa, Begingotra, RS d'Anjanaharibe-Sud, sur le versant Sud.Est, $2 \mathrm{~km}$ à 1'Ouest-Sud Ouest de Befingra, 1950-2000m, Racontodranairibe \& Raharimalala 2514 (P). Toliara: Andohahela RNI, Mont Trafanaomby, Parcelle I d'Andohahela Réserve Naturelle Intégrale, Tolagnaro, 1 000-1 957m, Randriamampionona 762 (P, UC). MALAWI: Mt. Mulanje, likhubula Valley, Chapalaka Trail, Chapman 7109 (UC 1587945). SUDÁFRICA: Transvaal: Woodbush Forest, Taylor 675 (UC 956260). Cape Town: Table Mountain, near Skeleton Gorge, Rodin 3211 (UC 802107). TANZANIA: Tanganiika: Irungi, Mufindi, N gwazi, Lovett \& Thompson 2381 (UC 1585473). ZIMBABUE: Orange Kop: Meltsetter, Wild 1953 (UC 085447). SUDAMÉRICA. ARGENTINA: Corrientes: Ituzaingó; desembocadura del arroyo Garapé en el río Paraná, $45 \mathrm{~km}$ al E de Ituzaingó, Schinini et al. 11239 (CTES). Misiones: Iguazú, Paraje Aguaray, Lote $\mathrm{P}$, bañado en rodal 5 y 6, sector anegado con Eryngium sp., Keller, Colcombet \& Ferreira 1774 (MA 710843). BOLIVIA: Santa Cruz: Ruinas Incaicas-El Fuerte, Steinbach 8259 (LIL 20684, planta de la derecha). BRASIL: Goiás: Serra do Cristais, $2 \mathrm{~km}$ by Road of Cristalina, elevat. 1200, Anderson 8148 (UC 1444720). Serra do Cristais, $2 \mathrm{~km}$ by Road of Cristalina, Anderson 6403 (P, UC). Minas Gerais: Sierra Cipó, Palacio Hotel, de la Sota 2616 (LIL 473356). Paraná: Curitiba, Colombo, Dombrovski 4442 \& Kuniyoshi 3620 (CTES, duplicados en MBM, PACA, citas de Dittrich 2005). Río de Janeiro: RS-Jaquirana, Fazenda Boa Vista, 900m, Watsum \& al. 3995 (MA 422162). Petropolis, Cuatrecasas 6137 (MA 233154, 9 pliegos). San Francisco de Paula, Villa Oliva, Rambo 31197 (LIL 206540 \& 182815). Serra do Itatiaia, Dussén 168 (P). Rio Grande do Sul: Bom Jesus, Dutra 278 (SI). s. loc. Jürgens 11 (SI). São Leopoldo, Rohr 280 (LIL 147188).
Santa Cruz, Rosenstock Filices Austrobrasiliensis 189, Juergens s. $n$. (LIL 474039). Santa Catarina: "Santa Catarina, nos campos", Reitz H680 (LIL146037). Bom Jardim, em bosques de Araucaria, Eskuche 0497 (CTES). Vienem bei Taquari, Serra do Mar, Ule 227 (P). São Paulo: Municipio Presidente Bernardes, Região do Pontal de Paranapanema, ca. 430m de alt., Rodovia SP-272 Pirapozinho-Mirante do Paranapanema, entre kms 22-26, Silva \& Fernandes 383 (CTES). Campos de Jordão, Leite \& Leite 3504 (LIL 162347). PARAGUAY: Alto Paraná, Reserva Biológica Limoy, Caballero Marmori 1597 (CTES). Caaguazú, Yhu, Sparre \& Vervoorst 2042 (LIL 382985). In regionis fluminis Alto Paraná, Fiebrig 5714 (LIL 41755). Guairá:Colonia Independencia, Schinini 1107 (CTES). In viam Caaguazú, Hassler 9389 (P). URUGUAY: Canelones, Bañados de Pando, Lombardo s. n. (LIL 206930). Bañados de Pando, Rosengurst B-4328 (LIL 15672). Bañados de Guazubirá, en la base de las dunas estables Carretera interbalnearia, Prada s. $n$ (MA). El Bañado de Los Pájaros, cerca de la carretera de Montevideo a Rocha, $p r$ Guazuvirá, Fuertes \& Prada s. n. (MA).

8. Blechnum werckleanum (H. Christ) C. Chr., Index filic.: 161. 1905. Lomaria werckleana H. Christ, Bull. Herb. Boissier, ser. 2, 4(11): 1091. 1904. Struthiopteris werckleana (H. Christ) Broadh., Bull. Torrey Bot. Club 39: 382. 1912. PROTÓLOGO: I. Wercklé 169. 1903. TIPO: Costa Rica, Wercklé 169 (US 575242-575243!; isótipo NY!).

Plantas terrestres con rizoma erecto de hasta $120 \mathrm{~cm}$ de alto, escamoso. Escamas rizomáticas lineares, de color castaño oscuro, rígidas, brillantes, curvadas todas en la misma dirección, hasta de $3.5 \mathrm{~cm}$ de largo. Frondas dimorfas, pinnadas, hasta de $180 \mathrm{~cm}$ de largo. Estípites rígidos adaxialmente surcados y con escamas basales similares a las rizomáticas. Láminas estériles oblongas, abruptamente reducidas en la base con algunas pinnas más cortas pero no vestigiales. Raquis y costas con escamas caducas pardo-amarillentas. Costas 
abaxialmente curvas y adaxialmente surcadas, con un surco poco profundo, con el haz vascular trabado por tejido parenquimático esclerosado que puede formar casquetes fibrosos independientes del haz. Pinna terminal conforme, en general más larga que las laterales, no adnata y prolongada en un ápice aserrado. Pinnas laterales 25-45 pares, herbáceas firmes, papiráceas y con márgenes rasgados cuando secas, distanciadas, oblongas angostas, ascendentes, sub-pecioluladas a pecioluladas, con ápice acroscópicamente curvado, caudado y aserrado, margen repando plano, abaxialmente más claras y cuando secas, con el envés pardoamarillento. Venas visibles, próximas, simples y bifurcadas una vez cerca de la costa, algunas geminadas, y siempre con una sola división en dos ramas claramente divergentes. Indusios eroso-fimbriados.

Distribución geográfica y ecología: Costa Rica, Panamá, Venezuela, Colombia, Ecuador, Perú. Selvas de neblina y páramos; 1000-2 500m de altitud.

Observaciones: Moran (1995) señala que B. werckleanum puede ser tanto terrestre como epífito, que carece de aeróforos y que la lámina seca es de color pardo oscuro en el haz y amarillo en el envés, algo que es útil en las identificaciones de material de herbario y que se ha comprobado aquí. Este autor sugiere que las plantas mesoamericanas podrían ser coespecíficas con B. schomburgkii, pero aquí se la trata como una especie característica, afín con B. moritzianum y con una distribución geográfica similar. Las pinnas discretas, ascendentes, curvadas acroscópicamente, distantes, subpecioluladas, discolores, con bases asimétricas y ápices caudados aserrados, difieren claramente de las pinnas de B. schomburgkii y son algo similares a las de $B$. moritzianum, aunque en esta especie son horizontales, más próximas, pecioluladas, con bases subcordiformes y ápices enteros agudos a subobtusos. En ambas las láminas de las pinnas están más desarrolladas que en otras especies arborescentes y los márgenes son planos. La confusión con $B$. schomburgkii es frecuente, tanto en el caso de $B$. werckleanum como de B. moritzianum.

Ejemplares de referencia seleccionados: COLOMBIA: Cauca: Macizo Colombiano, Páramo de Las Papas entre El Boquerón y La Hoyola, 3000-3500m, Hidrobo et al. 3451 (COL 64782). Cundinamarca: San Miguel, extremo SW de la Sabana de Bogotá, $2800 \mathrm{~m}$, Jaramillo Mejía, Murillo \& van der Hammen 3422 (MA 3480728). Cordillera Oriental, Páramo de Guasca, Laguna, 3200m, Cuatrecasas 2163 (MA 213337). Municipio de San Francisco, Vereda Sabaneta, Hacienda San Pedro, 2600-2800m, Medina \& Zamudio 90 (COL 253210). Huila: Comisaría del Caquetá, Cordillera oriental, sobre el filo divisorio, El Gabinete, 2300-2450m, Cuatrecasas 8490 (COL 14924). Nariño: Páramo El Consuelo, $10 \mathrm{~km}$ al $\mathrm{O}$ de la Victoria, $3100 \mathrm{~m}$, Hagemann \& Leist (COL 236369). PANAMÁ: Bocas del Toro: $1000 \mathrm{~m}$, Churchill et al. 4510 (UC 1506934). Chiriquí, along trail between North fork of Rio Palo Alto and Cerro Pate Macho, ca. 6km NE of Boquete, Smith et al. 2358 (UC 1524194). PERÚ: Cuzco: Paucartambo, 3350m, León \& Huallapa 2682 (USM). Piura: Huancabamba, above Huancabamba, road to Canchaque, 3 000m, Hutchinson 1620 (UC, USM 214614). VENEZUELA: Amazonas, Cerro de la Neblina, Camp 2, Neblina massif, 2.8km NE of Pico Phelps, 2700m, Stein \& Gentry 1537 (UC 1511485). Bolívar, Distrito Pilar, Camarcarbarai-tepui, summit 4th of 4 tepuis from $\mathrm{W}$ to $\mathrm{E}$ in Asparaman range, $2300 \mathrm{~m}$, Holst 3636 (UC 1535660).

\section{DISCUSIÓN}

Se ha realizado una revisión de ocho especies arborescentes de Blechnum: B. brasiliense, $B$. columbiense, B. cycadifolium, B. magellanicum, B. moritzianum, B. schomburgkii, $B$. tabulare y $B$. werckleanum. Todas estas especies tienen en común el hábito arborescente y las escamas aciculares, dos caracteres que originalmente se utilizaron para su agrupación (Tryon \& Tryon 1982), y el xilema de las costas 
en forma de $\mathrm{V}$ de brazos divergentes. Con excepción de $B$. brasiliense, también tienen en común las pinnas coriáceas, los haces costales trabados con esclerénquima, la presencia de 1-2 capas de hipodermis con células de paredes engrosadas, lignificadas o no, y las esporas con perisporio muriforme rugado, espaciadamente a densamente plegado, con muros bajos redondeados no anastomosantes.

Entre los caracteres estudiados relacionados con los ejes es destacable la constancia en la estructura vascular de las costas, diferente de la de otros grupos de especies afines (datos no publicados), constituida por un haz con xilema en $\mathrm{V}$ de brazos divergentes, rodeado por una endodermis y asociado con tejido esclerosado que forma una vaina perivascular que alcanza su mayor desarrollo (haces trabados adaxialabaxialmente) en especies que crecen entre 1200 y más de $3000 \mathrm{~m}$ (páramos, bosques fríos, bosques montanos tropicales) como $B$. columbiense, $B$. cycadifolium, $B$. magellanicum, $B$. moritzianum, $B$. schomburgkii, $B$. tabulare y $B$. werckleanum, mientras que falta en $B$. brasiliense, una especie que crece a menor altura en selvas lluviosas.

El desarrollo de este refuerzo de las pinnas podría estar relacionado con otro carácter no previamente estudiado en este grupo, que es la hipodermis. Esta capa dermatoide adaxial está formada por hasta tres capas de células dispuestas de manera continua. Las paredes celulares están lignificadas y las células son traqueiformes y largas en $B$. columbiense, $B$. cycadifolium y $B$. schomburgkii, mientras que no están lignificadas, son más cortas y tienen paredes menos engrosadas en $B$. magellanicum, $B$. moritzianum, $B$. tabulare y $B$. werckleanum, pero aún así contituyen un refuerzo mecánico continuo adicional para las pinnas. Las paredes de las células hipodérmicas son más delgadas que las de las epidérmicas en $B$. brasiliense. En todas las especies, con excepción también de $B$. brasiliense, se presenta además un mesofilo compacto.

Los modelos epidérmicos y la combinación de los tipos de epifilos e hipofilos son caracteres específicos, pero en los epifilos ciertas diferencias como profundidad y frecuencia de las sinuosidades, ondas simples o compuestas, engrosamiento parietal general o en las curvas y ángulos y la relación 1/a celular, son más evidentes por la ausencia de los estomas.

Los tipos esporales han sido analizados en detalle por Passarelli et al. (2010). Las esporas de la mayoría de las especies tienen perisporio plegado, con excepción de $B$. brasiliense que tiene perisporio venuloso. Esta especie también difiere del resto de los taxones arborescentes por las pinnas planas con márgenes aserrados y la presencia de protuberancias intercelulares pécticas en el parénquima que rodea al haz costal, aunque tiene en común el hábito, las escamas del rizoma y base del estípite y el tipo de haz costal. Las esporas de $B$. tabulare tienen una notable constancia en el área de la especie, con perisporios plegados y pliegues más bajos, menos evidentes que los que se presentan en las especies arborescentes andinas o de áreas templado-frías; las semejanzas con las esporas de $B$. madagascariense se señalaron previamente (Passarelli et al. 2010) al igual que las de B. schomburgkii y B. auratum subsp. auratum, aunque en ese trabajo se ilustraron separadamente, por estar por entonces inconcluso el estudio actual.

La clave para la determinación de las especies está basada en caracteres que se pueden apreciar en material vivo pero que se mantienen sin cambio en material de herbario y cuya apreciación no es difícil si se trabaja con material herborizado. Esto se ha hecho así porque a menudo este material es el único disponible para el botánico y en el caso particular de las especies arborescentes es escaso.

Blechnum schomburgkii y B. tabulare han sido revisados a la luz de nuevos enfoques de su morfología externa e interna, así como de su distribución geográfica, ecología y afinidades. Blechnum schomburgkii se considera aquí una especie preferentemente andina, presente en las laderas orientales de los Andes, los páramos y áreas montañosa de Venezuela y las Guayanas; es menos común en áreas selváticas del S-SE de Brasil, donde la especie se ha confundido con B. tabulare; es afín con B. moritzianum 
y $B$. werckleanum, dos especies que frecuentemente se determinan como B. schomburgkii en los herbarios, especialmente la primera. La variación morfológica que se podría atribuir a $B$. schomburgkii en función de las diferentes determinaciones halladas en material de herbario no es importante ni amplia si se la caracteriza como aquí se ha hecho, y se la diferencia de $B$. moritzianum y $B$. werckleanum. El análisis de los ejemplares de toda el área de distribución de B. schomburgkii ha permitido caracterizar de una forma más precisa las tres especies. En el caso de B. tabulare el análisis de ejemplares de toda su área geográfica ha llevado a reunir bajo ese nombre materiales que representaban parte de la variación morfológica de la especie y que se trataban como especies diferentes.

\section{AGRADECIMIENTOS}

Este trabajo fue realizado con el apoyo de las siguientes Instituciones: Ministerio de Ciencia e Innovación de España (Proyecto CGL 2009-13622, Subprograma BOS), Universidad Complutense de Madrid, España, Consejo Nacional de Investigaciones Científicas y Técnicas (CONICET), Buenos Aires y Programa de Incentivos para Docentes Investigadores de la Universidad Nacional de La Plata (Argentina). El Ministerio de Ciencia e Innovación de España apoyó la estadía de C. H. Rolleri en la Universidad Complutense de Madrid durante los meses de febrero a junio de 2010 a través de su Programa de Visitantes Distinguidos Universidad Complutense-Grupo Santander y la estancia de L.M. Passarelli en la Universidad Complutense en noviembre 2010. El Programa de Becas Complutense-del Amo financió una estancia de investigación de J.M. Gabriel y Galán en el Herbario de la Universidad de California en Berkeley. Se agradece la revisión crítica de aspectos nomenclaturales a Alberto Herrero, Real Jardín Botánico de Madrid, España.

\section{RESUMEN}

La bibliografía sobre el género Blechnum L. es muy abundante tanto en lo que respecta a las especies neotropicales como paleotropicales. Sin embargo, aún faltan estudios anatómicos completos, una caracterización actualizada de su morfología externa e interna, taxonomía, citología y afinidades. En este estudio se revisaron ocho especies de Blechnum de hábito arborescente: B. brasiliense, B. columbiense, B. cycadifolium, B. magellanicum, B. moritzianum, B. schomburgkii, B. tabulare y B. werckleanum. Se analizaron caracteres de rizomas, estípites y costas, división de la lámina, y venación, estructura de márgenes, modelos epidérmicos, presencia y tipos de hipodermis y mesofilo de las pinnas. Se estudió por primera vez la hipodermis, un área dermatoide adaxial presente en el mesofilo de todas las especies. Se correlacionaron caracteres externos e internos con el fin de utilizar los externos más confiables en claves y descripciones. Los estudios realizados confirmaron nuevamente la presencia de $B$. tabulare en América, que incluyen en su sinonimia a B. spannagelii de América tropical y B. madagascariense de África. También se concluyó que el nombre B. schomburgkii es un sinónimo de B. auratum subsp. auratum, en tanto que $B$. auratum subsp. columbiense se trata aquí con rango de especie. Se caracterizó a $B$. moritzianum, el nombre que corresponde aplicar a la recientemente descrita B. yungense. Se presentó una descripción actualizada de las especies, se resolvieron varios problemas nomenclaturales pendientes; se presentaron datos amplios sobre la ecología, distribución geográfica y afinidades entre las especies, así como una clave para determinarlas.

Palabras clave: revisión, especies arborescentes, morfología, nomenclatura, caracteres diagnósticos.

\section{REFERENCIAS}

Baeza, M., E. Barrera, J. Flores, C. Ramírez \& R. Rodríguez. 1998. Categorías de conservación de Pteridophyta nativas de Chile. Bol. Mus. Nac. Hist. Nat. 47: $23-46$.

Barrera, E. 1997. Helechos de Juan Fernández. Museo Nacional de Historia Natural, Publicación Ocasional $\mathrm{N}^{\circ} 51$.

Bower, F.O. 1923. The Ferns. Cambridge University, Cambridge, England.

Burrows, J.E. 1990. Southern African ferns and fern-allies. Fandsen, Sandton, South Africa.

Danton, P.H. 2004. Plantas silvestres de la Isla Robinson Crusoe, Guía de reconocimiento. Orgraf, Chile. 
Dittrich, V.A. 2005. Estudos taxonômicos no gênero Blechnum L. (Pteridophyta-Blechnaceae) para as regiões Sudeste e Sul do Brasil. Tesis de Doctorado, Universidad Estadual Paulista Júlio de Mesquita Filho, Rio Claro, São Paulo, Brasil.

Esau, K. 1953. Plant Anatomy. Wiley, Nueva York, EE.UU.

Font Quer, P. 1982. Diccionario de Botánica. Labor, Barcelona, España.

Foster, A.S. 1936. Leaf differentiation in Angiosperms. Bot. Rev. 24: 304-316.

Gabriel y Galán, J.M., L.M. Passarelli, C. Prada \& C.H. Rolleri. 2008. Study of sporophyte morphology and gametophyte development of Blechnum sprucei (Blechnaceae-Pteridophyta). Rev. Biol. Trop. 56: 2027-2040.

Hieronymus, G. 1908. Plantae Stübelianae. Hedwigia 47: 204-249.

Johow, F. 1896. Estudio sobre la Flora de las Islas de Juan Fernández. Cervantes, Santiago de Chile, Chile.

Lellinger, D.B. 2002. A Modern Multilingual Glossary of taxonomic Pteridology. Pteridologia 3: 1-246.

Metcalfe, C.R. \& L. Chalk. 1950. Anatomy of the Dicotyledons. Clarendon, Oxford, Inglaterra.

Moran, R.C. 1995. Blechnaceae, p. 325-333. In G. Davidse, M. Souza \& S. Knapp (eds.). Flora Mesoamericana I, Psilotaceae a Salviniaceae. Universidad Autónoma de México, Missouri Bot. Gard. \& The Nat. Hist. Museum, Londres, Inglaterra.

Moore, T. 1857. Index Filicum. W. Pamplin, Londres, Inglaterra.

Passarelli, L.M., J.M. Gabriel y Galán, C. Prada \& C.H. Rolleri. 2010. Spore morphology and ornamentation in the genus Blechnum (Blechnaceae). Grana 49: 243-262.

Payne, W.W. \& K.M. Peterson. 1973. Observations of the hypodermises of ferns. Am. Fern J. 63: 34-42.

Prabhakar, M. 2003. Structure, delimitation, nomenclature and classification of stomata. Acta Bot. Sin. 46: 242-252.

Prada, C. \& C.H. Rolleri. 2005. A new species of Isoetes (Isoetaceae) from Turkey, with a study of microphyll intercellular pectic protuberances and their potential taxonomic value. Bot. J. Linn. Soc. 147: 213-228.

Prada, C., C.H. Rolleri \& L.M. Passarelli. 2008. Morfología, caracterización y distribución geográfica de Blechnum cordatum (Blechnaceae-Pteridophyta). Acta Bot. Malac. 33: 29-46.

Ramos Giacosa, J.P. 2010. Blechnum yungense (Pteridophyta, Blechnaceae), una nueva especie de Argentina y Bolivia. Novon 20: 68-72.

Ramos Giacosa, J.P., M.A. Morbelli \& G.E. Giúdice. 2009 Spore morphology and wall ultrastructure of Blechnum L. species from North West Argentina. Rev. Palaeobot. Palynol. 156: 185-197.
Ricci, M. 1990. Programa de conservación y recuperación de plantas amenazadas de Juan Fernández. Informe Final, $2^{a}$ etapa, Proyecto CONAF-WWF-3313, Chile.

Ricci, M. 1992. Programa de conservación y recuperación de plantas amenazadas de Juan Fernández. Informe Final, $3^{\text {a }}$ etapa, Proyecto CONAF-WWF-3313, Chile.

Rodríguez Ríos, R. 1995. Pteridophyta, p. 119-309. In C. Marticorena \& R. Rodriguez (eds.). Flora de Chile. Vol. 1. Pteridophyta-Gymnospermae. Universidad de Concepción, Chile.

Rolleri, C.H. \& C. Prada. 2006a. Revisión de los grupos de especies del género Blechnum (Blechnaceae, Pteridophyta): el grupo Blechnum penna-marina. Acta Bot. Malac. 31: 7-50.

Rolleri, C.H. \& C. Prada 2006b. Catálogo comentado de las especies de Blechnum (Blechnaceae-Pteridophyta) de Mesoamérica y Sudamérica. Anales Jard. Bot. Madrid 63: 67-106.

Rolleri, C.H., C. Prada \& L.M. Passarelli. 2008. Estudios morfológicos y taxonómicos en Blechnum (Blechnaceae-Pteridophyta): Blechnum tabulare y B. magellanicum. Anales Jard. Bot. Madrid 65: 169-175.

Schelpe, E.A.C.L.E. 1952. A revision of the African species of Blechnum. J. Linn. Soc. Bot. 53: 487-510.

Skottsberg, C. 1924. The phanerogams of the Juan Fernández Islands, p 95-240. In C. Skottsberg (ed.). The Natural History of Juan Fernández and Easter Island, Vol II Botany. part VI. Almqvist \& Wiksells Boktryckeri AB, Uppsala, Suecia.

Skottsberg, C. 1953. The vegetation of the Juan Fernandez Islands, p. 793-959. In C. Skottsberg (ed.). The Natural History of Juan Fernández and Easter Island, Vol II. Botany. Part VI. Almqvist \& Wiksells Boktryckeri AB, Uppsala, Suecia.

Stolze, R.G. 1981. Ferns and fern allies of Guatemala. Part 2: Polypodiaceae. Fieldiana Bot. n.s. 6: 1-522.

Tardieu-Blot, M.L. 1955. Blechnum. In H. Humbert (ed.). Une merveille de la nature à Madagascar. Première exploration botanique du Massif du Marojejy et de ses satellites. Mém. Inst. Sci. Madagascar, Sér. B, Biol. Vég. 6: 230.

Tardieu-Blot, M.L. 1960. Blechnum. In H. Humbert (ed.). Flore de Madagascar et des Comores, 2: 1-19. Polypodiacees s. lat. (Blechnacees a Polypodiacees s.str.). Museum National d'Histoire Naturelle, Paris, Francia.

Tryon, R.M. \& A.F. Tryon. 1982. Ferns and Allied Plants with Special Reference to Tropical America. Springer, Nueva York, EE.UU.

Tryon, R.M. \& H. Lugardon 1990. Spores of the Pteridophyta. Springer, Nueva York, EE.UU.

Tryon, R.M. \& R.G. Stolze. 1993. Pteridophyta of Peru. Part. 5: Aspleniaceae-Polypodiaceae. Fieldiana Bot.n.s. 32: 54-70. 